\title{
Circuit
}

Musiques contemporaines

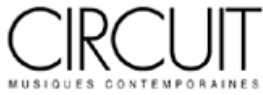

\section{La Seconde Apothéose de Rameau}

Au-delà de la polémique

\section{La Seconde Apothéose de Rameau (The Second Apotheosis of}

Rameau)

\section{Beyond the Polemic}

\section{Michel Gonneville}

Volume 12, numéro 1, 2001

Henri Pousseur : visages

URI : https://id.erudit.org/iderudit/902239ar

DOI : https://doi.org/10.7202/902239ar

Aller au sommaire du numéro

Éditeur(s)

Les Presses de l'Université de Montréal

ISSN

1183-1693 (imprimé)

1488-9692 (numérique)

Découvrir la revue

Citer cet article

Gonneville, M. (2001). La Seconde Apothéose de Rameau : au-delà de la polémique. Circuit, 12(1), 44-71. https://doi.org/10.7202/902239ar
Résumé de l'article

Analyse technique détaillée, assortie de nombreux exemples musicaux et tableaux, de l'oeuvre de Pousseur composée en 1981. L'oeuvre est reliée à un texte polémique de 1968 sur la question harmonique et à un argument programmatique évoquant musicalement cette polémique. Construite comme une succession de citations et d'autocitations intimement imbriquées et ici identifiées, La Seconde Apothéose de Rameau est ainsi vue comme l'emblème du postmodernisme musical. L'auteur cherche également à constamment mettre en rapport l'analyse technique avec une appréciation esthétique de l'oeuvre. 


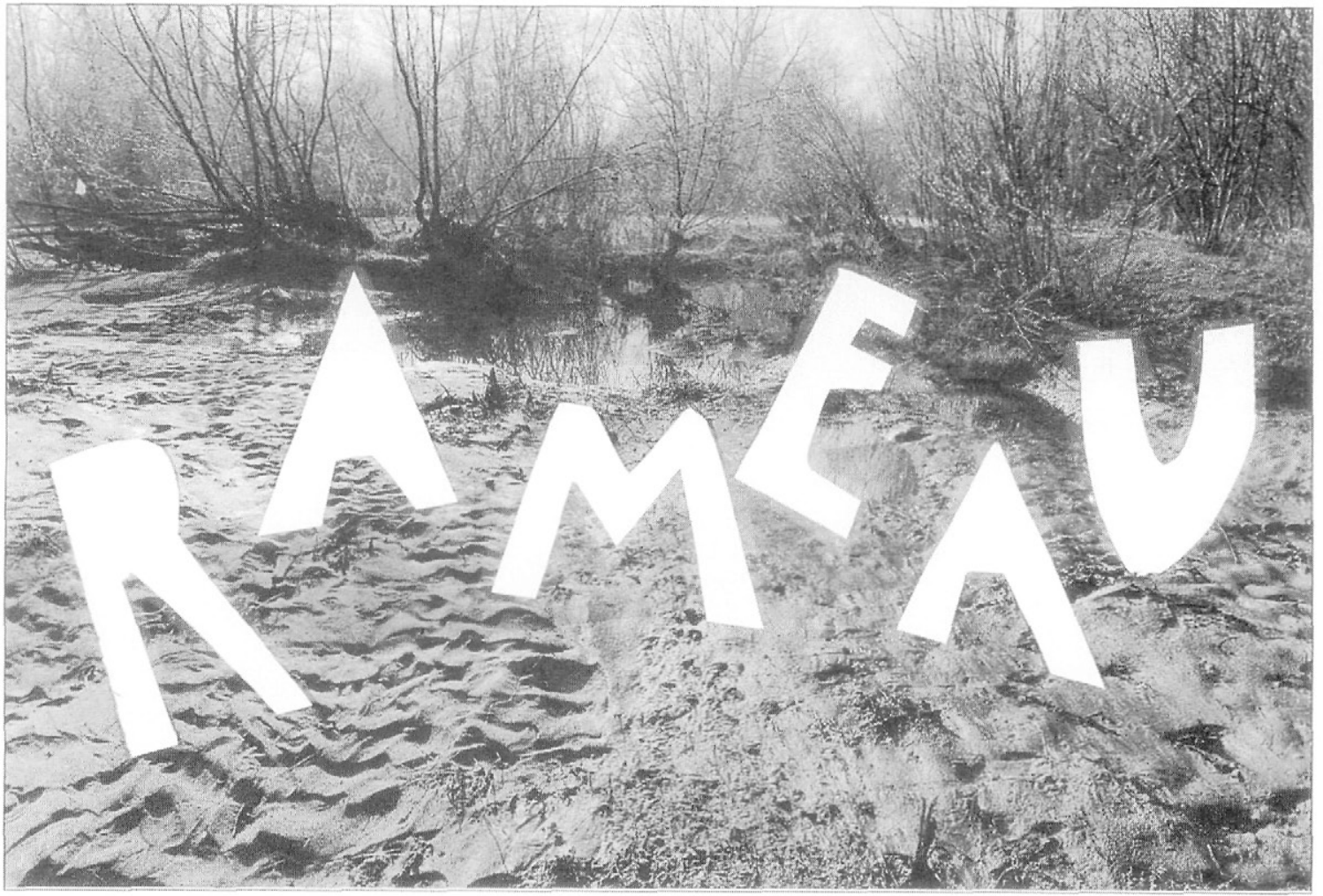

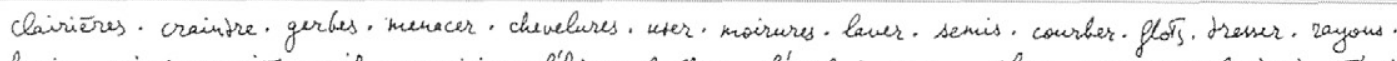
bruire, airsi courrait auril poursuivi par l'hiver. halliers. l'aigle du casque. vibarer, comme une broderie. Témoins.

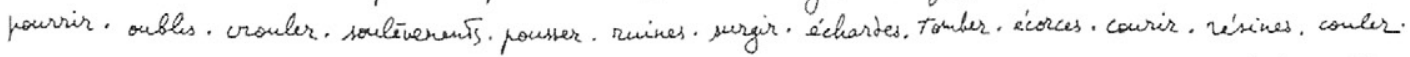
sucled $r$ 


\section{La Seconde Apothéose de Rameau : au-delà de la polémique}

Michel Gonneville

\section{Introduction : une œuvre postmoderne}

Pour qui s'intéresse (encore...) au postmodernisme - dont l'éclectisme est devenu un peu superficiellement un concept à la mode et presqu'un slogan, autant sociopolitique qu'artistique - , parmi les quelque 150 œuvres du catalogue d'Henri Pousseur, La Seconde Apothéose de Rameau (1981) pourrait bien faire figure d'emblème. On y trouverait plusieurs des traits définissant ce "mouvement", surtout du point de vue du rapport à l'histoire /citations, intégration de mondes harmoniques-stylistiques différents voire ambigus), et jusqu'à cette ironie ludique caractéristique de l'«attitude postmoderne».

N'oublions pas cependant qu'à la date de composition de cette œuvre, nous avons déjà derrière nous Hymnen (1966) de Stockhausen et Sinfonia (1968) de Berio, qui sont de grands classiques du collage, en plus d'une partie très éloquente des travaux de Cage, Kagel et Ligeti, compositeurs qui sont très vite allés au-delà des limitations "modernistes" dictées et imposées par l'époque. À cette date également, Pousseur s'est déjà précédé lui-même let peut-être a-til été l'inspirateur du mouvement de dépassement chez ses confrères célèbres) en composant des œuvres modèles comme Votre Faust (1961-1968), Couleurs croisées (1967), les Éphémérides d'lcare II (1970) et son clone lcare apprenti (1970), Vue sur les jardins interdits (1973), le théâtre musical Die Erprobung des Petrus Hebraicus (1974) et la symphonie stylistique Les Îles déchaînées (1980). Mais l'œuvre de 1981 a ceci de particulier qu'elle permet de renover avec le point de départ, tant musical qu'idéologique, de cette tendance de fond chez lui (depuis Votre Faust) et d'insister sur la pertinence de cette approche, puisqu'elle s'est même répandue chez quelques-uns des jeunes loups de l'époque. Elle permettait peut-être aussi, subtile- 
ment, d'organiser, au moins sur le plan symbolique et pour soi, une "réparation" pour l'incompréhension (même silencieuse) subie alors. Mais laissons Pousseur nous situer lui-même en citant in extenso les notes de programme de l'œuvre.

Écrite en 1968, la première Apothéose de Rameau était un long texte théorique, destiné à expliciter (dans son "pourquoi" et son "comment") la réactualisation de fonctions harmoniques (par exemple les "consonances") rigoureusement exclues par les musiques sérielles jusqu'à cette date (pour des raisons momentanément excellentes). Cette réactualisation n'avait pas été entreprise dans un but rétrospectif ni en tout cas "néo-classique", mais au contraire pour arriver à une "sérialité élargie", proprement généralisée. La fin de l'article, qui justifiait la dédicace du texte à Pierre Boulez, indiquait également qu'un jour, ce même titre pourrait bien être celui d'«un morceau de musique».

Cette promesse fut enfin réalisée près de dix ans plus tard, à l'occasion d'une demande faite par Pierre Bartholomée l pour le concert qu'il devait diriger à I'Intercontemporain, à l'automne de 1981. Et si un temps si long s'était écoulé entre les deux travaux, leur propos me semblait rester suffisamment constant pour qu'entre eux (et entre les deux domaines: théorie et invention musicale) je puisse établir une sorte d'homothétie.

Celle-ci se construit autour d'une trame principale fortement directionnelle: le "Prologue dans le ciel» de Votre Faust, premier exemple réussi (pour moi! en 1963) d'un passage graduel entre dissonance chromatique et consonance diatonique let retourl, est présent en deux versions, plus ou moins variées dans leur "épaisseur»; quant à la Vue sur les jardins interdits, à l'origine pour quatuor de saxophones (1973) mais dont furent ensuite rédigées cinq autres versions (pour orgue, quatuor à cordes, trio à cordes, etc.l, et qui suit le même chemin circulaire mais en se déduisant d'une citation de Samuel Scheidt et mettant donc davantage l'accent sur l'aspect conducteur des linéaments polyphoniques, elle est exactement préservée dans son texte musical, et seulement réorchestrée pour le plus large effectif disponible (neuf bois, sept cuivres et cinq instruments à sons amortis ${ }^{2}$. Ces trois séquences formelles sont subdivisées en de nombreux fragments, interpolées en alternance pour ne plus former qu'un seul flux, partant du plus "contemporain "(typiquement post-webernien) pour y revenir à la fin, mais après avoir creusé une perspective historique de plus en plus profonde, particulièrement accusée par la présence de nombreuses citations parfois beaucoup plus brèves, greffées sur ce tronc principal et soudant entre eux ses tronçons constitutifs : qui vont de Stravinsky et Schönberg, en passant par Webern, jusqu'à Boulez, Stockhausen, Berio, voire Pousseur (exemples tirés du texte théorique) et Bartholomée eux-mêmes. Le moment "tabernaculaire» marqué par l'apparition avouée du vieil organiste allemand (Scheidt), évoque aussi assez longuement Rameau, comme il se doit, auréolé par l'hommage que lui a rendu Debussy.

Tout ce propos quasi narratif lqui raconte effectivement une histoire : la nôtre, à la fois musicale et polémiquel est résumé de manière fabulée dans l'«Argument» que voici, joint à la partition :
1. Chef d'orchestre et compositeur, Pierre Bartholomée est l'ami et le complice de longue date de Pousseur. Il a été, entre autres, directeur musical de l'Orchestre phitharmonique de Liège et fondateur de l'ensemble Musique nouvelle de liège.

2. Xylomarimba, vibraphone, harpe lamplifiée), piano électrique et célesta, piano. Les percussionnistes jouent aussi de quelques autres instruments à hauteurs indéterminées. 
1. Au Parnasse, des anges novices que leur état incite à la modestie et dont le nom ne sera dès lors pas prononcé, discutent avec feu des vertus d'un ancestral rameau et de l'utilité d'encore récolter ses fruits.

2. Cependant l'archange Antoine réitère les leçons de l'ancien jardinier, tout en prouvant par des travaux originaux que leur application pratique peut naturellement être bien plus diversifiée que celle faite en son temps.

En conséquence, les séraphins Arnold et lgor visitent de concert la retraite du vieil ermite pour le persuader d'oublier l'aveuglement de précédentes générations et le prier d'assister à la fête que l'on projette en son honneur.

3. Dès avant leur retour, le bienheureux Claude-Achille prépare un hommage circonstancié, dans lequel il proposera, pour réparer les ingratitudes perpétrées à l'égard du saint et assurer qu'elles ne puissent se reproduire, d'« écarteler son visage " aux dimensions du firmament.

\section{La polémique : où nous mène Pousseur?}

Le mot a été lâché : La Seconde Apothéose de Rameau sera donc une polémique en musique où citations et autocitations vont incarner en quelque sorte les personnages de leurs créateurs et les faire ainsi dialoguer entre eux. Le "supercompositeur " qui les organise a tout de même le gros bout du bâton puisqu'il pourra même, comme on le verra, pousser Boulez et Stockhausen à se plier à ses "arguments" et à se métamorphoser de façon bien conciliante... II pourra également mettre en scène, à son profit, l'appui de quelques grandes figures historiques. Mais l'œuvre ainsi écrite constituera surtout une nouvelle invitation à passer outre aux interdits que l'avant-garde s'imposait (et s'impose peut-être encore) à elle-même, afin d'aller au cœur même des raisons (philosophiques et esthétiques, sociologiques, politiques) de l'attitude moderne, plutôt que d'en rester à considérer ses traits de surface (grammaire, langage, style).

Dans cet article, nous chercherons à faire ressortir les grandes lignes de ce qui constitue le nœud de cette polémique musicale, le point de départ de cette discussion, en nous concentrant sur le plan harmonique ${ }^{3}$.
3. L'analyse qui va suivre a été entreprise avec un groupe d'étudiants de la classe d'analyse (niveau DESM II) du Conservatoire de musique de Montréal, lesquels en ont présenté les premiers résultats (déjà bien avancés) au public du séminaire d'analyse dans le cadre de l'événement "Visages d'Henri Pousseur ». Je tiens à remercier pour leur travail : Alain Beauchesne, Christian Gort et Mathieu Marcoux, qui ont eu à se concentrer sur les composantes de la Seconde Apothéose de Rameau, de même que Julien Bilodeau, Nicolas Gilbert ef Parrick Saint-Denis qui ont apporté leur contribution aux recherches.

\section{Analyse des composantes de ce collage :}

L'armature principale de la Seconde Apothéose de Rameau est composée, rappelons-le, de deux autocitations de Pousseur. 


\section{Autocitation I: «Prologue dans le ciel» de Votre Faust}

Survenant tout de suite après la scène parlée initiale de Votre Faust ${ }^{4}$, lle "Prologue sur le théâtre»|, cette courte pièce instrumentale constitue pour Pousseur, comme il le mentionnait dans ses notes de programme, "le premier exemple réussi d'un passage graduel entre dissonance chromatique et consonance diatonique (et retour)". L'article de 1968 fait longuement état des techniques qui auront été utilisées dans cette pièce, de même que des explorations qui y auront mené. On pourra compléter les informations données ici par celles de l'article de Pascal Decroupet (1989). Rappelons ces données brièvement.

Neuf séries de douze sons (mais ne contenant pas nécessairement le total chromatique!) servent à fournir une suite de notes principales (ou notes génératrices). Ces séries sont les suivantes : celle des Chants sacrés de Pousseur lui-même, celle, très proche des Zeitmaße de Stockhausen, celle des Structures de Boulez, celle de la Cantate $n^{\circ} 2$ de Webern - transposition du rétrograde -, suivie de trois transformations de cette dernière ${ }^{5}$, une série du Canticum sacrum de Stravinsky, et enfin celle du Trio à cordes de Schönberg. Les séries sont transposées de façon à s'enchaîner par note commune, la dernière note d'une série étant la première de la suivante (exemple 11. On notera que les séries transformées obtenues à partir de celle de Webern, de même que celle de Stravinsky, ne contiennent que dix sons différents de la gamme chromatique et possèdent des inflexions diatoniques assez remarquables.
4. Sur Votre Faust, « fantaisie variable de genre opéra " qu'Henri Pousseur a écrit en collaboration avec l'écrivain Michel Butor, une abondante littérature existe, depuis les multiples versions du livret, parues dans les années 60 à la NRF jusqu'aux nombreuses études sur l'œuvre et ses implications.

5. Grâce aux pistes données par Pousseur (1968 et communication par courriel), il nous a été possible d'élucider la filiation entre la série originale de Webern et ses trois transformations, de même que celle de la série de Stravinsky dans le Canticum sacrum. L'exposé du mécanisme de ces transformations déborde cependant le cadre de cet article.

\section{Exemple 1 : les 9 séries de notes génératrices}

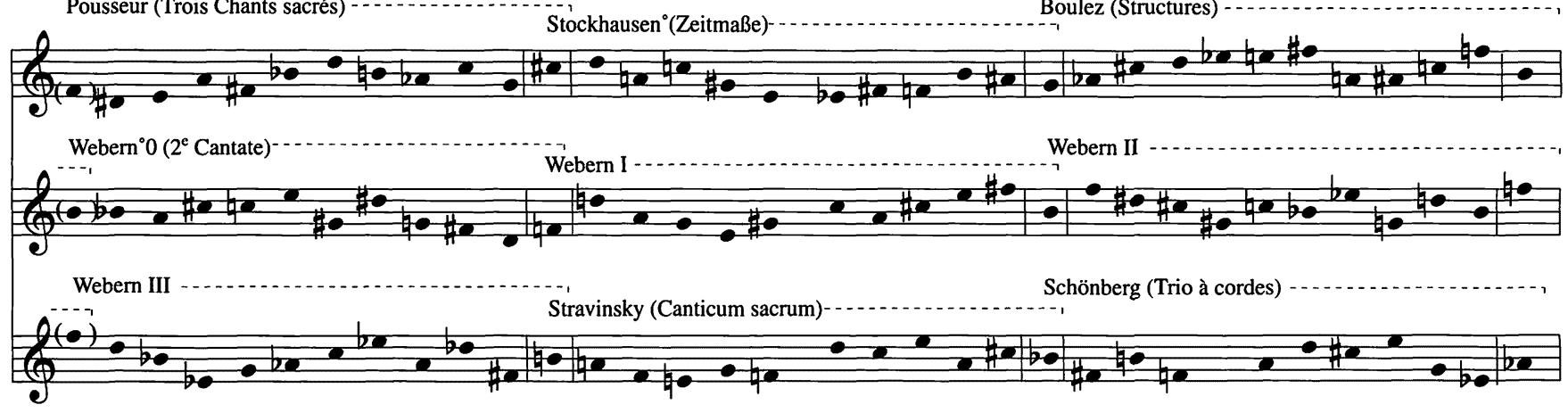


Sur chaque note génératrice viennent se greffer un certain nombre de notes secondaires formant un "groupe" avec celle-ci. Beaucoup plus que la séquence des notes génératrices, ce sera celle des notes secondaires, successives ou simultanées, tout autant que leur registration, qui seront déterminantes pour la perception d' "atmosphères" harmoniques différentes. Parmi les trois techniques régissant l'engendrement de ces notes secondaires, la principale consiste en deux cycles de quintes imbriqués dont l'un effectue une rotation sur lui-même par rapport à l'autre. L'exemple 2 donne la liste des douze séries de 24 sons issues de ce procédé. Pousseur ayant déjà commenté amplement les propriétés des séries ainsi obtenues, on se bornera ici à faire remarquer que, de par la logique des rotations, les séries centrales 6 et 7 rejoignent le total chromatique très rapidement lau bout de 12 ou 13 sons successifs) alors que les séries extrêmes (1 et 12) ne le font qu'au bout des 24 sons, présentant (si l'on prend des groupes de 11 ou 12 sons successifs de ces séries) un diatonisme qui évolue lentement dans le courant de la série.

Exemple 2 : douze séries de 24 sons obtenues par 2 cycles de quintes imbriqués dont l'un en rotation

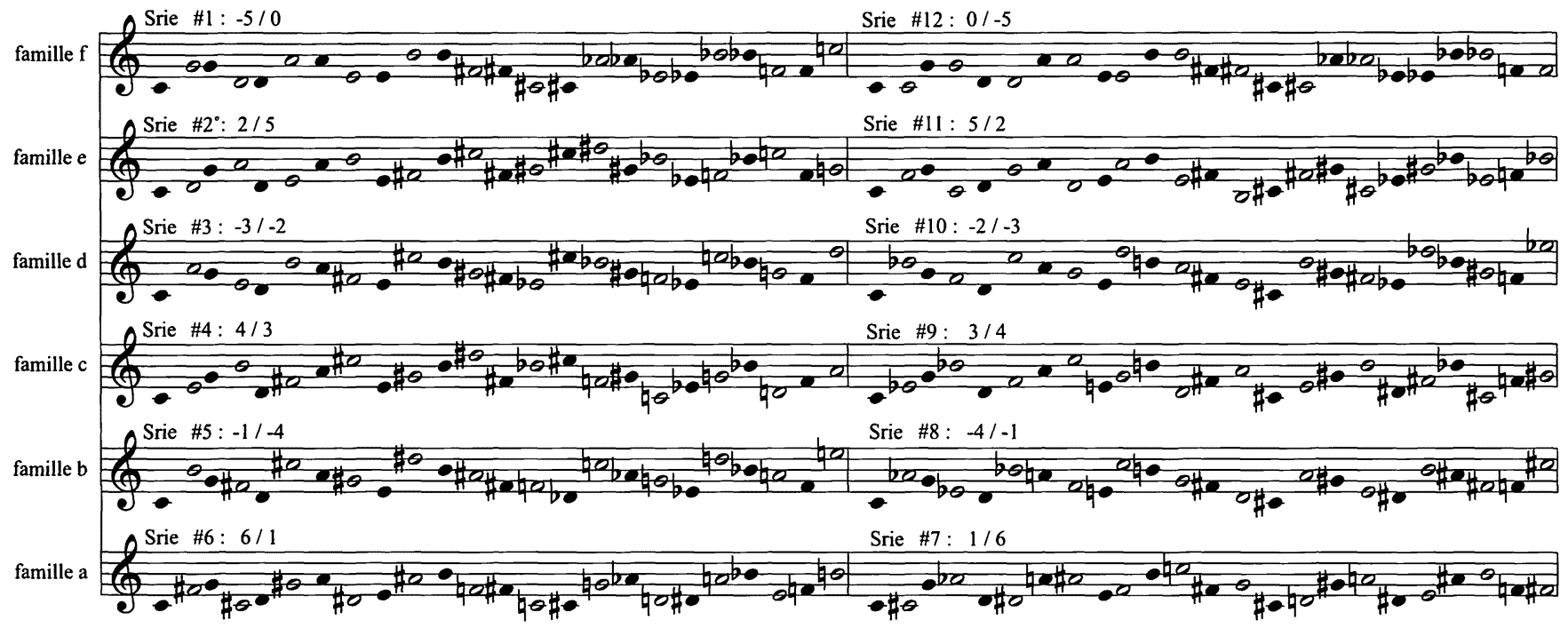

Une seconde technique, utilisée entre les mesures 84 et 96 du "Prologue», divise la gamme chromatique en deux gammes par tons imbriquées et également en rotation l'une par rapport à l'autre. L'exemple 3 donne deux de ces rotations; toutes donnent le total chromatique en 12 notes. On doit aussi se souvenir que toutes les séries ainsi obtenues peuvent être lues de façon renversée ou rétrograde. 
Exemple 3 : séries obtenues par 2 gammes par tons imbriquées dont l'une en rotation

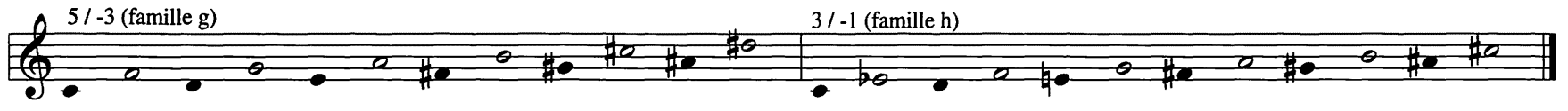

On aura remarqué que toutes ces séries sont constituées par la répétition cyclique de deux intervalles; les différentes séries peuvent donc être résumées et désignées par ces deux intervalles ${ }^{6}$ (donnés pour chaque série dans les deux exemples précédents). Selon le critère de similarité des intervalles générateurs, nous avons regroupé les 12 premières séries en 6 familles désignées par les lettres a à $f$; les deux séries engendrées selon la seconde technique sont désignées par les lettres $g$ et $h$. La dernière technique, utilisée pour les mesures 90 et 97 à 102 , est essentiellement de type chromatique et distribue - d'une manière, peutêtre sérielle, que nous n'avons pu élucider - les notes d'un cluster chromatique, les différents résultats étant tous considérés de famille harmonique $i$.

Les notes secondaires sont donc engendrées à partir de la note génératrice, en suivant la logique (ou le cycle) intervallique de la série en cours. L'exemple 4 donne quelques mesures extraites du "Prologue (réduit sur deux portées) illustrant chacune un type harmonique différent. La portée inférieure donne la note génératrice (boules blanches) de même que les notes secondaires engendrées par les deux intervalles générateurs. La mesure 67 donne un exemple de permutation dans l'ordre des notes qui arrive parfois, associé souvent à des jeux de symétrie distributionnelle. II est aussi assez fréquent de trouver (particulièrement dans les zones des séries de Webern) une répartition des notes du groupe sous forme de blocs (accords) symétriques (comme à la mesure 26).

On remarquera que, quelle que soit la technique utilisée pour les engendrer, le nombre de notes secondaires attachées à une note génératrice correspond toujours à celui qui est nécessaire pour que la note génératrice suivante soit rejointe à la fin des notes secondaires, en suivant la logique du cycle intervallique. Dans le cas de la technique chromatique, ce nombre correspond tout simplement à l'intervalle à franchir en demi-tons (par exemple: sixte majeure $=9$ demi-tons $=9$ notes dans le groupe $=$ note génératrice +8 notes secondaires).

Le tableau 1 présente de façon synoptique, mesure par mesure, les notes génératrices et leurs séries d'appartenance, les intervalles générateurs servant à l'engendrement des notes secondaires, de même que les regroupements des groupes en ensembles ("phrases " $A$ à $W$ ) séparés par des silences, représentés par des tirets $(-)$, qui les délimitent ainsi et les définissent.

Quant à l'organisation formelle du plan harmonique, une lecture attentive de ce tableau permet de remarquer :

- I'association à peu près constante d'une famille d'intervalles générateurs avec une série génératrice : Pousseur : famille $a$; Stockhausen : famille $b$
6. Notés selon la technique habituelle, selon le nombre de demi-tons contenus par l'intervalle : $0=$ unisson, $1=$ seconde mineure, 2 = seconde majeure, 3 = tierce mineure, 4 = tierce majeure, 5 = quarte juste, $6=$ triton, chaque intervalle pouvant être affecté d'un signe - (moins) s'il est descendant, ce qui permet de couvrir les renversements des intervalles précédents, depuis la quinte juste jusqu'à l'octave $(-5=7,-4=8,-3=9$, $2=10,-1=11,0=121$.

Cette technique de cycle intervallique contient en germe la future technique des réseaux que Pousseur développera par la suite et dont l'article de 1968 commente l'application dans Couleurs croisées pour orchestre, datant de 1967. 
Exemple 4 : quelques extraits du Prologue dans le ciel
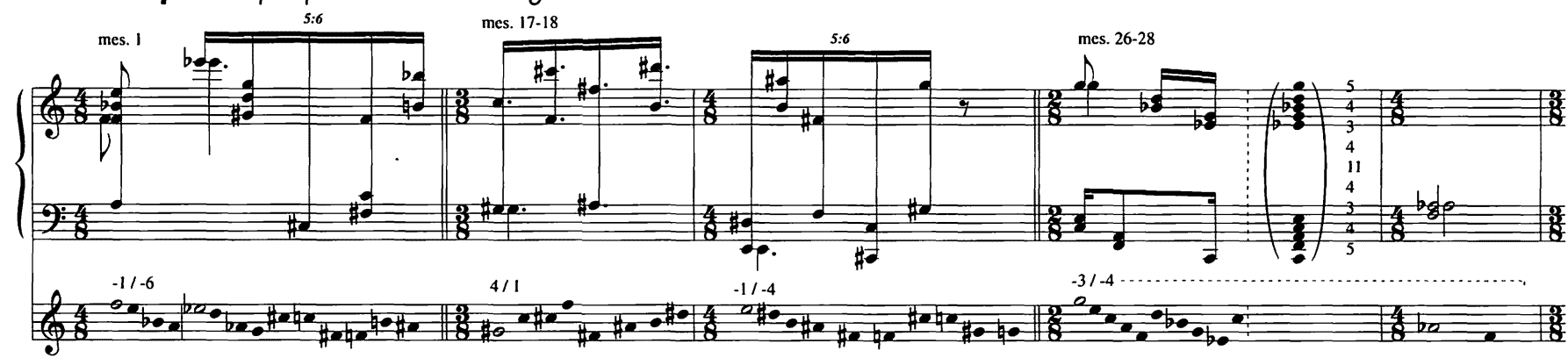

$-1 /-4$

$-3 /-4$
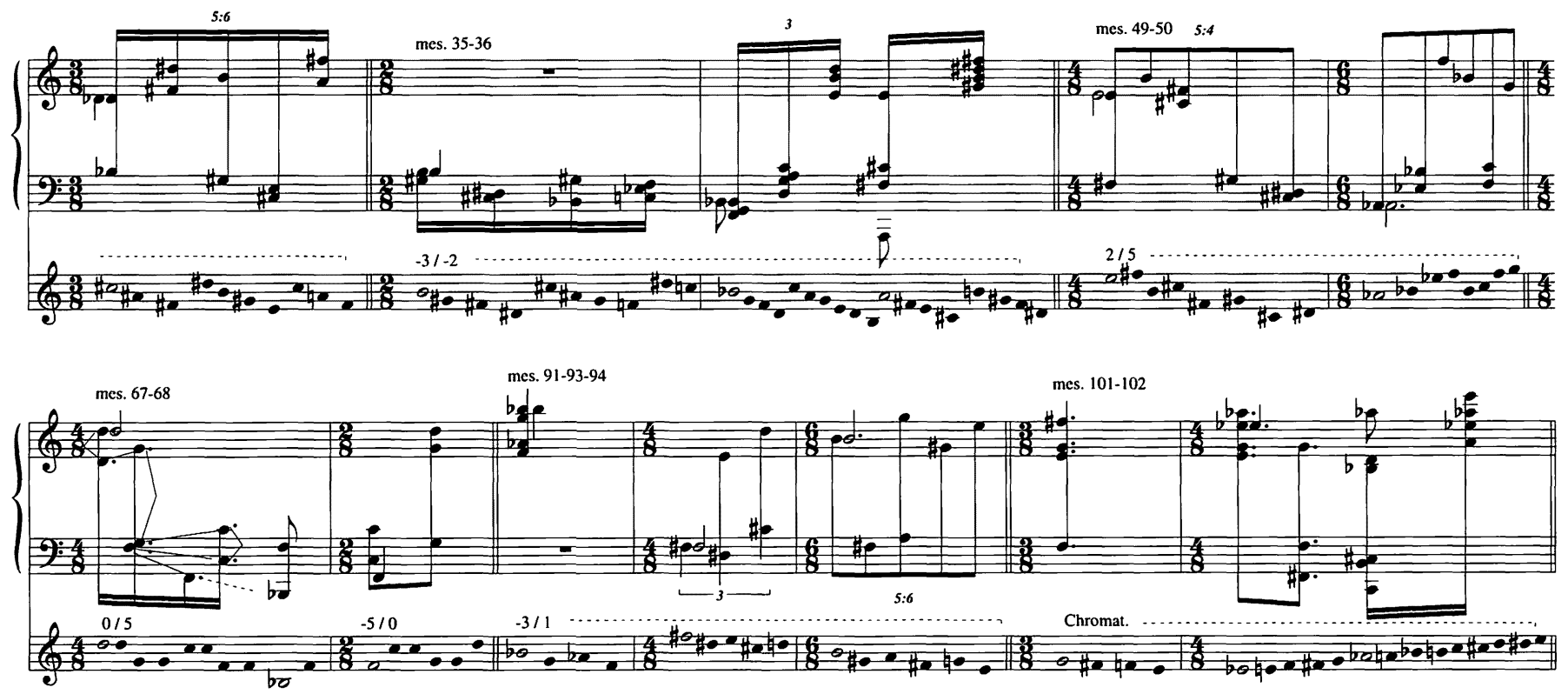

(avec retours à al; Boulez: famille $c$; Webern 0 : famille $d$; Webern I: famille e (avec un retour à $d$ ): Webern $I$ et $I I I$ : famille $f$ lavec un retour à $c$ ): Stravinsky: familles $a$, puis $b$, puis enfin $g$ (avec une annonce de il; Schönberg : familles $h$ et $i$ (chromatique). Cette association non seulement ne coïncide pas nécessairement avec la structure des "phrases", mais elle n'a aussi que peu à voir avec les intervalles dominants de ladite série, sinon que les familles d'intervalles générateurs les plus consonants coïncident avec les trois transformations de la série de Webern, aux inflexions fortement diatoniques.

- la courbe générale, précédemment soulignée, allant progressivement d'ensembles généralement "dissonants" et dépolarisés vers un sommet de 
Tableau 1 : tableau synoptique des ensembles, groupes, notes génératrices et intervalles générateurs dans Prologues dans le ciel deVotre Faust

\begin{tabular}{|c|c|c|c|c|c|c|c|c|c|c|c|c|c|c|c|c|c|c|c|c|c|c|c|c|c|c|c|c|c|}
\hline Mesures & 1 & & 2 & 3 & 4 & 5 & & 6 & & 7 & 8 & 9 & 10 & 11 & 12 & 13 & 14 & 15 & 16 & 17 & 18 & & 19 & 20 & 21 & 22 & 23 & 24 & 25 \\
\hline Durée du groupe (en croches à 120) & 1 & 3 & 3 & 2 & 4 & 1 & 3 & 7 & 2 & 2 & 4 & 4 & 6 & 4 & 2 & 4 & 2 & 2 & 4 & 3 & 3 & 1 & 2 & 4 & 4 & 4 & 5 & 3 & 3 \\
\hline Ensemble de groupe (<phrases $\gg \mid$ & A & & & & & & & - & $B$ & & - & $\mathrm{C}$ & & - & $D$ & & & - & $E$ & & & - & $F$ & & & - & $G$ & & - \\
\hline Série notes principoles / familles harmoniques & Pouss & seur $/$ & & & & & & & & & & & & & Stockl & hause & & b & & & & & & & & & & & \\
\hline Notes principoles & $\mathrm{Fo}$ & Mib & $\mathrm{Mi}$ & La & Solb & Sib & Ré & - & $\mathrm{Si}$ & Sol\# & - & $D_{0}$ & Sol & - & Do\# & Ré & 10 & - & Do & Sol\# & $\mathrm{Mi}$ & - & Mib & Fa\# & $\mathrm{Fo}$ & - & $\mathrm{Si}$ & Sib & - \\
\hline $\mathrm{I}^{\text {en intervalle du cycle des intervalles générateurs }}$ & 1 & 1 & -1 & 6 & 6 & 6 & 6 & $\bar{z}$ & 6 & 6 & - & 6 & -1 & - & 4 & -1 & 4 & - & 4 & 4 & -1 & - & 4 & -1 & (4) & - & 6 & 6 & - \\
\hline $2^{\mathrm{e}}$ intervalle du cycle des intervalles générateurs & 6 & 6 & 6 & 1 & 1 & 1 & 1 & - & 1 & 1 & - & 1 & 6 & - & 1 & -4 & 1 & - & 1 & 1 & -4 & - & 1 & -4 & (II) & - & 1 & 1 & - \\
\hline Nombre de notes dans le groupe & 4 & 10 & 2 & 6 & 8 & 8 & 6 & - & 6 & 8 & - & 2 & 12 & - & (111) & 2 & 6 & - & 8 & 8 & 10 & - & & $|110|$ & 12 & - & 9 & (6) & - \\
\hline
\end{tabular}

\begin{tabular}{|c|c|c|c|c|c|c|c|c|c|c|c|c|c|c|c|c|c|c|c|c|c|c|c|c|c|c|c|c|c|c|c|c|}
\hline esures & 26 & 27 & 28 & 29 & 30 & 31 & & 32 & & & 34 & 35 & 36 & & 37 & & & 40 & 41 & 42 & & \begin{tabular}{|l|}
43 \\
\end{tabular} & 44 & 45 & & 46 & & 47 & 48 & & 50 & \\
\hline urée & & 4 & 3 & 3 & 3 & 2 & 1 & 4 & 3 & 1 & 2 & 2 & 1 & 1 & 3 & 3 & 7 & 4 & $5 \mid$ & 1 & 2 & 3 & 2 & 2 & 1 & 3 & & 4 & 6 & 4 & 6 & 2 \\
\hline 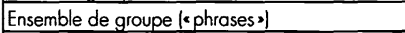 & $\mathrm{H}$ & & & & & & & & & & & & & & & & & - & 1 & & & & & & & $\mathrm{~J}$ & & & & $\mathrm{~K}$ & & \\
\hline érie r & Boul & $\mathrm{ez} / \mathrm{s}$ & & & & & & & & & & Web & bern 0 & & & & & & & & & & & Web & bern & $\mid / \mathbf{e}$ & Caussi & $i: d$ & a 531 & & & \\
\hline Vole & Sol & Lab $/ R$ & Réb & Ré & $\mathrm{Mib}$ & $\mathrm{Mi} F$ & Fa\# & La & Sib & Do & $\mathrm{Fa}$ & $\mathrm{Si}$ & Sib & La & Do\# & Do & $\mathrm{Mi}_{\mathrm{i}}$ & - & Lab & Mib & Sol & Fa\# & Ré & $\mathrm{Fa}$ & - & Ré & La & Sol & - & $\mathrm{Mi}$ & Lab & \\
\hline inte & -3 & -3 & -3 & -3 & -3 & 4 & -3 & -3 & 4 & -3 & 4 & -3 & -3 & -3 & -3 & -3 & -3 & - & -3 & -3 & -3 & 2 & 2 & 2 & - & 2 & -5 & 2 & - & 2 & 2 & - \\
\hline intervalle du cycle des intervalles & -4 & -4 & -4 & -4 & -4 & 3 & -4 & -4 & \begin{tabular}{|c|c|c|}
3 & -1 \\
\end{tabular} & -4 & 3 & -2 & \begin{tabular}{|l|}
-2 \\
\end{tabular} & -2 & -2 & \begin{tabular}{|l|} 
\\
\end{tabular} & -2 & - & $-2 \mid$ & -2 & -2 & 3 & 3 & 5 & - & 5 & -2 & 5 & - & 5 & 5 & - \\
\hline lombre de note dans le groupe & 10 & 2 & 10 & 10 & & 4 & 6 & 10 & & 2 & 12 & 11 & & & & & & & 2 & & 0 & & & & & & & & & & & \\
\hline
\end{tabular}

\begin{tabular}{|c|c|c|c|c|c|c|c|c|c|c|c|c|c|c|c|c|c|c|c|c|c|c|c|c|c|c|c|c|c|c|c|}
\hline esures & 2 & 53 & 54 & 55 & 56 & 57 & 58 & 59 & & 60 & & 61 & 62 & 63 & 64 & 65 & 66 & & 67 & & 68 & 69 & & 70 & 71 & 72 & & 73 & 74 & 75 & 76 \\
\hline urée du groupe len croches à $120 \mid$ & 8 & 2 & 6 & 2 & 2 & 4 & 3 & 3 & 1 & 3 & 1 & 4 & 2 & 6 & 2 & 8 & 2 & 1 & 3 & 1 & 2 & 2 & 1 & 3 & 5 & 1 & 1 & 3 & 3 & 3 & 4 \\
\hline sembl & & & - & $M$ & & - & $N$ & & - & 0 & & & & & - & $\mathrm{P}$ & & & & & & & & & & & & & & & \\
\hline principales / familles h & & & & & & & & Web & pern & $1 / \mathrm{f}$ & & & & & & & & & & & Web & ern III & $/ f \mid c$ & aussi & $\grave{\mathrm{a}}$ & & & & & & \\
\hline tes & Do & La & - & Do\# & $\mathrm{Mi}$ & - & Fa\# & $\mathrm{Si}$ & - & Solb & Mib & Réb & Lab & Do & - & Sib & Mib & Sol & Ré & Sib & $\mathrm{Fa}$ & Ré & Sib & Mib & Sol & Solft & Do & & Lab & Réb & Fa\# \\
\hline inte & 2 & -3 & - & -5 & 2 & - & -5 & 2 & - & -5 & 0 & -5 & -5 & 0 & - & 0 & (10) & -5 & 0 & -5 & -5 & 0 & 0 & 0 & 10) & -4 & (10) & 0 & 0 & 0 & 0 \\
\hline intervalle du cycle des intervalles générateurs & 5 & -2 & - & -2 & 5 & - & -2 & 5 & - & 0 & 5 & 0 & 0 & 5 & - & 5 & $\mid 15)$ & 0 & 5 & 0 & 0 & 5 & 5 & -5 & (5) & -3 & (5) & 5 & 5 & 5 & 5 \\
\hline ombre de nole dans le groupe & 6 & (8) & - & 6 & 4 & - & 2 & 2 & $1-$ & 5 & & & & & - & 2 & & & & & 6 & 8 & & & & & & & & & \\
\hline
\end{tabular}

\begin{tabular}{|c|c|c|c|c|c|c|c|c|c|c|c|c|c|c|c|c|c|c|c|c|c|c|c|c|c|c|c|c|c|c|c|}
\hline \begin{tabular}{|l|} 
Mesures \\
\end{tabular} & 77 & 78 & 79 & 80 & 81 & 82 & 83 & 84 & & 85 & 86 & 87 & \begin{tabular}{|l|}
88 \\
\end{tabular} & 89 & \begin{tabular}{|l|}
90 \\
\end{tabular} & 91 & 92 & 93 & 94 & 95 & 96 & 97 & & 98 & & & 1001 & 101 & 102 & & \\
\hline Durée du groupe len croches a 120) & 2 & 3 & 3 & 2 & 4 & 6 & 8 & 2 & 1 & 3 & 3 & 2 & 2 & 4 & 6 & 2 & 4 & 4 & 6 & 4 & 4 & 2 & 1 & 3 & 1 & 4 & 2 & 3 & & 1 & \\
\hline Ensemble de groupe («phroses $\curvearrowright \mid$ & & - & $Q$ & & & - & $R$ & & - & $\mathrm{s}$ & & & & - & $\mathrm{T}$ & & - & $\mathrm{U}$ & & - & $\mathrm{V}$ & & - & $\mathrm{W}$ & & & & & & & \\
\hline Série notes principales / familles harmoniques & Strav & insk & Ig & lous: & si : $\mathrm{f}_{\text {, }}$ & $a, b$ & $i$ et $x$ & & & & & & & & & Schö & inber & $g / h$ & et $\mathbf{i}$ & $\mid=$ chr.. & & & & & & & & & & & \\
\hline Noles principales & $\mathrm{Si}$ & - & la & Fa\#t & $\overline{\mathrm{Mi}}$ & - & Sol & $\mathrm{Fa}$ & - & Ré & Do & $\mathrm{Mi}$ & la & -1 & Do\# & $\mathrm{Sib}$ & - & Fa\# & $\mathrm{Si}$ & - & $\mathrm{Fa}$ & Do & - & La & Rél & Do\# & $\mathrm{Mi}$ & Sol & Mib & Lab & $(\mathrm{fal})$ \\
\hline $\mathrm{I}^{e r}$ intervalle du cycle des intervalles générateurs & 0 & - & (16) & (16) & $|-1|$ & - & \pm 4 & 5 & - & |51 & 5 & (6) & 5 & - & Chr. & -3 & - & -3 & -3 & - & 3 & Chr. & - & Chr. & Chr.f & Chr. & Chr. & Chr. & Chr. & Chr. & \\
\hline $2^{\mathrm{e}}$ intervalle du cycle des intervalles généraleurs & 5 & - & $(-1)$ & (III) & $(-4)$ & - & \pm 3 & -3 & - & $(-3)$ & -3 & (5) & -3 & - & & 1 & - & 1 & 1 & - & -1 & & - & & & & & & & & \\
\hline Nombre de nole dans le groupe & 4 & - & 7 & 9 & (9) & - & 110 & 5 & - & 110 & 4 & 11 & 4 & - & 11 & 4 & - & 5 & of & - & 5 & & - & 5 & 12 & & 9 & 4 & 5 & 9 & \\
\hline
\end{tabular}

consonance (coïncidant avec les séries Webern II et III), puis un retour vers le chromatisme, d'abord brutal $|a|$, puis amorti par des familles intermédiaires $(g$ et $h)$.

On verra plus loin comment le "Prologue " est utilisé dans La Seconde Apothéose de Rameau. II sera alors utile de se rappeler que l'articulation musicale générale reste constamment disjointe, les notes étant distribuées aux différents instruments à la manière de la Klangfarbenmelodie webernienne, par points ou courtes cellules également disjointes sur le plan mélodique, cette disjonction spatiale et timbrale étant amplifiée par ailleurs, sur le plan temporel, par la subdivision du morceau complet en "phrases» séparées par des silences. C'est d'ailleurs la chose la plus saisissante que d'entendre l'évolution harmonique mentionnée ci-haut dans le contexte d'une 
telle articulation. Si les premières mesures font très "musique contemporaine», très vite l'harmonie de plus en plus bouscule en renouvelle les stéréotypes.

\section{Autocitation II : Vue sur les jardins interdits}

Cette autocitation crée déjà une mise en abîme puisqu'elle contient en ellemême, voire est engendrée par, la citation d'une œuvre de Samuel Scheidt, une courte chanson ${ }^{7}$ :
7. La forme $A A^{\prime} B A$ de cette pièce se détaille ainsi : $a b$ a' $b^{\prime} c d a b$. Les membres $a^{\prime}$ ef $b^{\prime}$ constituent uniquement une harmonisation légèrement différente des membres a

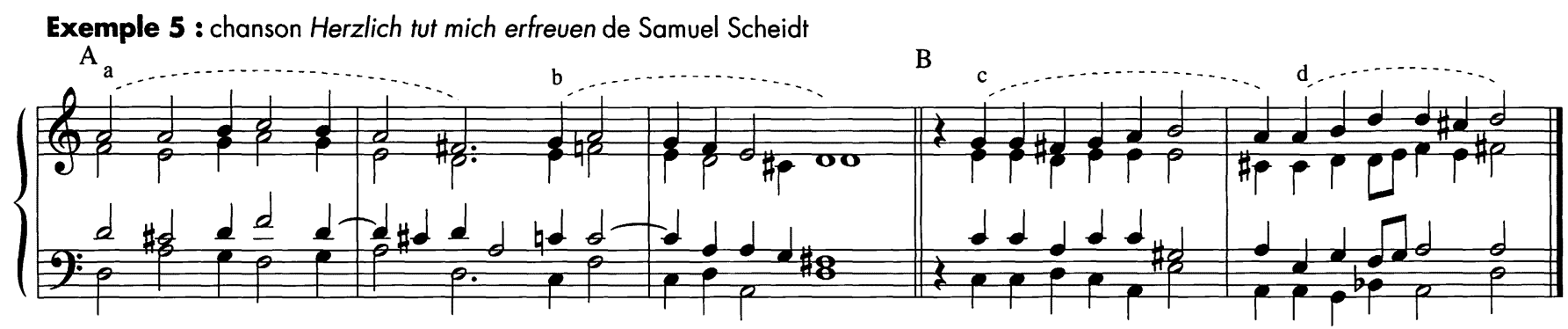

Encore une fois, citons les notes de programme écrites par Henri Pousseur :

Vue sur les jardins interdits a été composé à l'automne de 1973 [et] est dédié à la mémoire de Bruno Maderna, mort pendant sa composition. Une chanson de Samuel Scheidt (XVIr siècle), à mi-chemin entre le choral et la danse, et composée essentiellement de divers enchainements d'accords parfaits, est entendue au centre de la forme, dans une version déjà légèrement amplifiée /du point de vue de l'extension tonale). Elle sert de cellule génératrice à toutes les autres sections qui en constituent les variantes, déformations, anamorphoses - d'autant plus poussées qu'elles sont plus éloignées de ce noyau, vers le début ou vers la fin. De telle sorte que l'on perçoit essentiellement une texture moderne (commençant et se terminant par une modulation sonore proche de phénomènes électroacoustiques) au cœur de laquelle se creuse peu à peu l'espace nécessaire à la citation, toute naturelle, véritablement organique, de l'ancestrale polyphonie.

Comme nous l'avons dit plus haut, nous nous attacherons peu dans cette analyse à l'aspect textural, c'est-à-dire à tout ce qui caractérise les relations entre eux des instruments du quatuor de saxophones (hétérorythmie, filtrages d'accords, regroupements en duos), donc à tout ce qui varie l'écriture principalement homophonique de cette œuvre. Nous ne nous attarderons pas non plus aux caractères qui naissent et $b$ (avec un peu plus d'accords renversés), et, à ce que nous sachions, Pousseur ne se sert pas de ces variantes comme point de départ pour son travail harmoniquel. 
de l'action conjuguée du rythme, de l'intensité et de l'articulation. Tous ces jeux sont pourtant beaucoup plus variés ici que dans le "Prologue», et la dramatique de la musique en est conséquemment plus animée et contrastée. Nous nous concentrerons de nouveau sur la progression harmonique et pour pouvoir la suivre plus facilement, nous avons constitué un tableau (exemple 6) regroupant par familles tous les accords utilisés dans Vue ${ }^{8}$. Pour constituer ces familles, nous avons procédé selon une technique qui s'apparente à la set-theory de Forte et alii.
8. Nous n'avons pas vérifié auprès d'Henri Pousseur la pertinence poiétique lou génétique) de cette classification en familles harmoniques, mais elle s'est révélé malgré cela un outil descriptif tout à fait adéquat.

Exemple 6 : accords de base pour Vue sur les jardins interdits, dans l'ordre de leur entrée.

(20) (10)

Comme toute l'œuvre, même avec ses variations texturales, peut être ramenée à des enchaînements d'accords de quatre sons, nous avons réduit tous les accords de l'œuvre en regroupant leurs notes constitutives dans le plus petit intervalle possible, et les groupes de quatre notes ainsi obtenus ont ensuite été transposés de façon à tous avoir le do central comme note la plus grave. Les 41 familles d'accords de l'exemple 6 sont ainsi apparues. Sous l'accord de base de chaque famille sont écrits les trois intervalles successifs qui décrivent cet accord de base. L'exemple 7, qui donne quelques extraits de Vue mis en regard de leur analyse harmonique, permet de comprendre concrètement cette méthode. Ainsi les quatre notes du tout premier accord de la pièce (mes. $\left.1-1-1^{9}\right)$ comprend les notes fa - fa dièse - sol - la bémol qui, ramenés sur do, correspondent aux quatre notes de l'accord de base de la famille 1 dans l'exemple 6.
9. Pour régler le problème de la numérotation des mesures, l'œuvre comportant des passages non mesurés assez longs, nous avons d'abord convenu de subdiviser ceuxci à chacune des respirations signifiée par une virgule léventuellement surmontée d'un point d'orguel de même qu'aux barres de mesures en lignes pointillées, puis (pour diminuer l'ambiguité causée par l'imprécision éventuelle de cette méthodel de désigner les mesure par une série de trois nombres séparés par des tirets et désignant successivement la page de la partition, le système (il y en deux par page) et la mesure dans ce système. 
Exemple 7 : quelques passages extraits de Vue sur les jardins interdits ef leur analyse harmonique
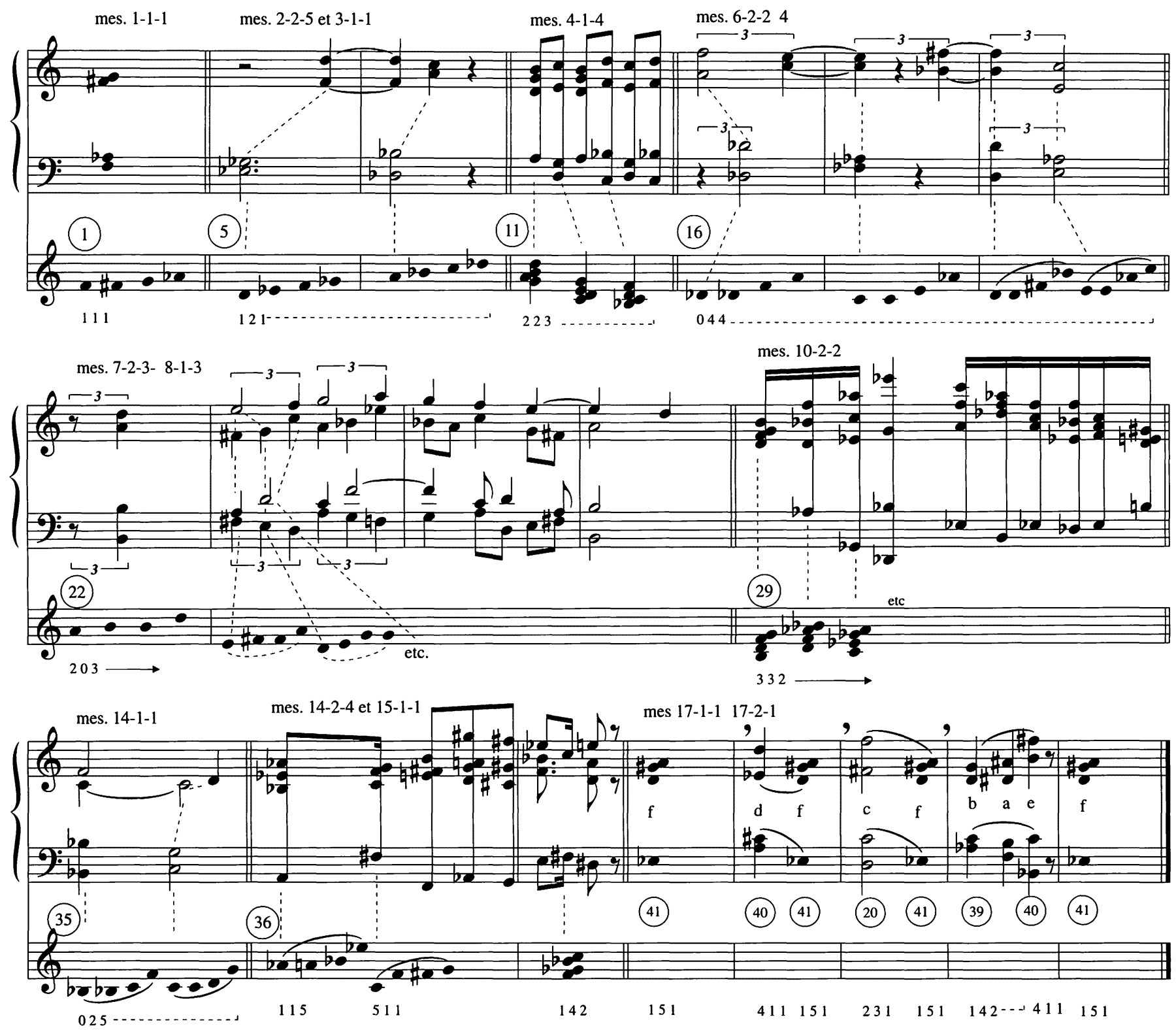
Dans l'exemple 6, les accords de base des 41 familles sont présentés là un détail près) dans l'ordre de leur apparition au cours de l'œuvre. Cette présentation permet d'appréhender, hors contexte, la grande courbe harmonique de l'œuvre ${ }^{10}$. Ainsi, pour le début de Vue, ce seront les familles plus chromatiques qui domineront (1 à 6,9 et, à un degré moindre, 7). Puis les familles plus diatoniques entrent en jeu $(8,10,11,13)$ avec un passage par un groupe par tons (12). De 14 à 17, les familles qui se succèdent ont un accord de quinte augmentée pour tronc commun, et la quatrième note de l'accord contrecarre une tendance vers la gamme par tons inhérente à cet accord augmenté (familles 14 et 17), ou alors y contribue (familles 15 et 16 ). Cette couleur de gamme par tons se retrouve également dans la famille suivante (18). Après des familles de transition 119 et 20 plus chromatiques, 22 et 23 plus diatoniques, 24 et 25 plus ambiguës), commence la série des familles ayant l'accord parfait majeur comme tronc commun (26 à 32), à une exception près (30). C'est dans ce groupe de familles que s'insérera la citation de Scheidt, comme il se doit, dont il a été dit qu'à peu près tous ses accords constitutifs sont des accords parfaits majeurs à l'état fondamental (famille $\left.32(33)^{\prime \prime}\right)$. Après la citation, les familles 34 et 35 vont ensuite vers une consonance encore plus grande, vers les quartes, quintes et octaves. Puis les familles finales, 36 à 41 , redeviennent brusquement beaucoup plus chromatiques.

II ne faut pas oublier que nous parlons ici des couleurs d'accords individuels, pris séparément, et dont la succession pourra éventuellement amplifier ou perturber, selon le cas, la perception d'un mode que leur structure pourrait appeler ${ }^{12}$.

Le tableau 2 permet de voir de façon plus détaillée la progression harmonique que nous venons de survoler, avec les fréquents retours en arrière et la prégnance réelle des familles dans le temps (ne serait-ce qu'au point de vue du nombre de mesures). Un passage particulièrement intéressant à observer est celui dont on vient de parler, qui présente les accords dont le tronc commun est l'accord parfait majeur. Si l'on considère la succession réelle des familles de cette section (mes. 92-1 à 13-1-4), en y incluant le retour des familles 14 et 11 l) et en supprimant la famille 30 dont l'emploi est très passager (1 accord!), on obtient la succession suivante de familles : $26,27,14,28,29^{13}, 11,31,32$. En lisant cette sucession à la lumière de l'exemple 6 , on aura la surprise d'y constater que les notes étrangères ajoutées à cet accord-tronc (do majeur) se succèdent de façon à former là une déviation près) une portion de cycle de quintes se dirigeant peu à peu vers le redoublement de la fondamentale de cet accord: fa dièse, ré bémol, la bémol, ré dièse, si bémol, (ré), fa, do. C'est à ce dernier point, précisément qu'arrive la citation intégrale de Scheidt, entre 11-1-3 et 13-1-4! Les familles d'accords mentionnées, qui précèdent la citation, auront toujours placé la note étrangère à la basse (comme une "fausse basse"), les trois voix supérieures disposant l'accord parfait de différentes façons (voir, dans l'exemple 7, l'extrait provenant de la mesure 10-22). On aura aussi noté que déjà à compter de 9-2-4, des annonces de la chanson de Scheidt sont interpolées (famille 32), et lorsque celle-ci s'installe, des persistances de 31 la crible encore quelque temps ${ }^{14}$.
10. On se référera avantageusement, pour ce qui suit, aux extraits de l'exemple 7, qui présente des illustrations pour les familles 1 , $5,11,16,22,29,35$ et 36 .

11. L'inclusion d'accords mineurs dans la chanson de Scheidt oblige à inclure la famitle de cet accord, mais sa présence faible nous autorise à le mettre entre parenthèses (33), accompagnant toujours 32 .

12. Ainsi, la succession chromatique d'accords augmentés annihilerait la perception potentielle de la gamme par tons inhérente à la structure de l'accord augmenté.

13. La logique de notre mode de représentation a malheureusement le défaut de présenter l'accord de septième naturelle (famille 29) sous forme renversée.

14. Beaucoup pourrait être dit sur cette pièce : sur l'association des accords d'une famille avec un caractère musical ou une texture particulière; sur le caractère synthétique des accords finaux, associant les demi-tons des familles initiales aux quartes et quintes de celles qui suivent la citation (34 et 35); sur le rapport des différentes sections du morceau à la chanson de Scheidt là ce propos, on pourra examiner l'extrait des mesures 7-2-3 à 8-1-3, dont la basse reproduit, en séquence puis de façon rétrograde, un passage de la basse de la chanson), etc. 
Tableau 2 : plan formel synoptique présentant la succession des familles d'accords de base dans Vues sur les jardins interdits

\begin{tabular}{|c|c|c|c|c|c|c|c|c|c|c|c|c|c|c|c|c|c|c|c|c|c|c|c|c|c|c|c|}
\hline Numéro de page et de système & \multicolumn{5}{|c|}{$1-1$} & \multicolumn{4}{|c|}{$1-2$} & \multicolumn{4}{|c|}{$2-1$} & \multicolumn{5}{|c|}{$2-2$} & \multicolumn{5}{|c|}{$3-1$} & \multicolumn{4}{|c|}{$3-2$} \\
\hline numéro de mesure & 1 & 2 & 3 & 4 & 5 & 1 & 2 & 3 & 4 & 1 & 2 & 3 & 4 & 1 & 2 & 3 & 4 & 5 & 1 & 2 & 3 & 4 & 5 & 1 & 2 & 3 & 4 \\
\hline numéro de famille harmonique & 1 & & & & & & & 1 & 2 & 3 & 4 & 2 & 5 & & 2 & 6 & & 5 & & 3 & 4 & 7 & & 5 & 8 & & $6-9$ \\
\hline Segmentation de la pièce & 1 & & & & & $\|$ & & & & III & & & & & & & & IV & & & & & & & V & & \\
\hline
\end{tabular}

\begin{tabular}{|c|c|c|c|c|c|c|c|c|c|c|c|c|c|c|c|c|c|c|c|c|c|c|c|c|}
\hline Numéro de page et de système & $4-1$ & & & & $4-2$ & & & & $5-1$ & & & & $5-2$ & & & $6-1$ & & & $6-2$ & & & & & 7-1 \\
\hline numéro de mesure & 1 & 2 & 3 & 4 & \begin{tabular}{|l|l|}
1 & 2 \\
\end{tabular} & 3 & 4 & 5 & 1 & 2 & 3 & 4 & 1 & $2 \mid 3$ & \begin{tabular}{l|l}
3 & 4 \\
\end{tabular} & 1 & 2 & 3 & 12 & 3 & 4 & 5 & $6: 1$ & 1 \\
\hline numéro de famille harmonique & & 10 & 5 & 11 & \begin{tabular}{|l|l|}
8 & 12 \\
\end{tabular} & 13 & & & & 12 & & & 10 & 14 & & 11 & 15 & 11 & 16 & & & 15 & & 17 \\
\hline Segmentation de la pièce & & & & & $\mathrm{VI}$ & & & & & & & & VII & & & & & VIII & & & & & & \\
\hline
\end{tabular}

\begin{tabular}{|c|c|c|c|c|c|c|c|c|c|c|c|c|c|c|c|c|c|c|c|c|c|c|}
\hline \multicolumn{5}{|l|}{ Numéro de page et de système } & \multicolumn{3}{|c|}{$7-2$} & \multicolumn{4}{|l|}{$8-1$} & \multicolumn{3}{|l|}{$8-2$} & \multicolumn{4}{|c|}{$9-1$} & \multicolumn{4}{|l|}{$9-2$} \\
\hline numéro de mesure & 2 & 3 & 4 & 5 & 1 & 2 & 3 & 12 & \begin{tabular}{|l|l|}
3 & 4 \\
\end{tabular} & 5 & 6 & \begin{tabular}{l|l}
1 & 2 \\
\end{tabular} & \begin{tabular}{|l|l|}
2 & 3
\end{tabular} & 4 & 1 & $2 \mid 3$ & \begin{tabular}{l|l}
3 & 4 \\
\end{tabular} & 5 & \begin{tabular}{l|l}
1 & 2 \\
\end{tabular} & 3 & 4 & 5 \\
\hline numéro de famille harmonique & 18 & 19 & & & 20 & 21 & 19 & 22 & 23 & 248 & 2311 & 24 & & 8 & & 25 & & & 26 & & & 27 \\
\hline Segmentation de la pièce & & IX & & & $x$ & $\mathrm{XI}$ & XII & & XIII & & & XIV & & $X V$ & & $X V I$ & & XVII & $X V I I I$ & & XIX & \\
\hline
\end{tabular}

\begin{tabular}{|c|c|c|c|c|c|c|c|c|c|c|c|c|c|c|c|c|c|c|c|c|c|c|c|c|c|}
\hline \multirow{2}{*}{$\begin{array}{l}\text { Numéro de page et de système } \\
\text { numéro de mesure }\end{array}$} & \multirow[b]{2}{*}{6} & \multicolumn{3}{|c|}{$10-1$} & \multicolumn{4}{|c|}{$10-2$} & \multicolumn{6}{|c|}{$11-1$} & \multicolumn{6}{|c|}{$11-2$} & \multicolumn{4}{|c|}{$12-1$} & \multirow{2}{*}{\begin{tabular}{|l|}
122 \\
\end{tabular}} \\
\hline & & 1 & 2 & 3 & 11 & $2 \mid 3$ & $3 \mid 4$ & 45 & 1 & 2 & 3 & 4 & 5 & 6 & 1 & 2 & 3 & 4 & & 6 & 1 & 2 & 3 & & \\
\hline numéro de famille harmonique & & (32) & 142 & 28 & (32) 2 & $29 \mid 3$ & 32) 1 & 1 & & 031 & 32 & & (31) & & & & (31) & 321 & 33) & & & & & & \\
\hline Segmentation de la pièce & & & & $x X$ & & & & & & & & & & & & & $\mathrm{XXI}$ & & & & & $X X I I$ & & XXIII & XXIV \\
\hline
\end{tabular}

\begin{tabular}{|c|c|c|c|c|c|c|c|c|c|c|c|c|c|c|c|c|c|c|c|}
\hline \multicolumn{5}{|c|}{ Numéro de page et de système } & \multicolumn{3}{|l|}{$13-1$} & \multicolumn{3}{|c|}{$13-2$} & \multicolumn{3}{|l|}{$14-1$} & \multicolumn{3}{|l|}{$14-2$} & \multicolumn{3}{|c|}{$15-1$} \\
\hline numéro de mesure & 2 & 3 & 4 & 5 & \begin{tabular}{|l|l|}
1 & 2 \\
\end{tabular} & \begin{tabular}{|l|l|}
3 & 4 \\
\end{tabular} & 5 & 1 & 2 & 3 & \begin{tabular}{l|l|}
1 & 2 \\
\end{tabular} & \begin{tabular}{|l|l|}
3 & 4 \\
\end{tabular} & 5 & \begin{tabular}{l|l}
1 & 2 \\
\end{tabular} & 3 & 4 & 1 & 23 & \begin{tabular}{l|l}
3 & 4 \\
\end{tabular} \\
\hline \multicolumn{8}{|c|}{ numéro de famille harmonique } & 34 & $32: 333^{2}$ & $23 \mid 3$ & \begin{tabular}{|l|l|}
35 & 30 \\
\end{tabular} & \multicolumn{3}{|l|}{31} & $29-28$ & \multicolumn{2}{|c|}{$36(39)$} & \multicolumn{2}{|c|}{31} \\
\hline Segmentation de la pièce & & $X X$ & & $X X$ & & XXVII & & $\mathrm{XVIII}$ & $X X X$ & $X X X$ & & $X X X \mid$ & & & $X X X \mid 1$ & & & & XXXIII \\
\hline
\end{tabular}

\begin{tabular}{|c|c|c|c|c|c|c|c|c|c|c|c|c|c|c|c|c|c|c|c|}
\hline \multicolumn{2}{|l|}{ Numéro de page et de système } & \multicolumn{4}{|c|}{$15-2$} & \multicolumn{3}{|l|}{$16-1$} & \multicolumn{6}{|c|}{$16-2$} & \multicolumn{4}{|c|}{$17-1$} & \\
\hline numéro de mesure & 5 & 1 & 2 & 3 & 4 & 1 & 2 & 3 & 1 & 2 & 3 & 4 & 5 & 6 & 1 & 2 & 3 & & \\
\hline numéro de famille $h$ & 17 & & 37 & 38 & $-31-17$ & & & $\begin{array}{l}39 \\
a b\end{array}$ & $a b$ & aba & $\begin{array}{c}20 \\
c\end{array}$ & $\begin{array}{l}\text { 40-39 } \\
\text { d ba }\end{array}$ & $\begin{array}{r}40 \\
e\end{array}$ & $\begin{array}{l}20-40 \\
\text { c d }\end{array}$ & $\begin{array}{c}41 \\
f\end{array}$ & $d f$ & cf & bae & \\
\hline Segmentation de la pièce & & & $X X X$ & & $X X X V$ & XXXVI & $X X$ & $\lambda V$ & & & & $X X X V \|$ & & XXXIX & XL & & & & \\
\hline
\end{tabular}




\section{Utilisation des deux autocitations : armature de La Seconde Apothéose.}

Pour constituer l'armature de La Seconde Apothéose de Rameau, le "Prologue" est fragmenté selon les phrases A à W figurant au tableau 1 et Vue selon les segments I à XL du tableau 2. Les deux morceaux sont évidemment réorchestrés pour l'ensemble disponible. L'utilisation du "Prologue" a ceci de particulier qu'elle fait avancer en parallèle deux versions de celui-ci, l'une souvent plus élaborée et démultipliée que l'autre.

On pourra examiner cette mise en place des fragments du "Prologue» et de Vue dans le tableau 3 qui présente une vue synoptique de la Seconde Apothéose. Les indices + et - qui suivent les lettres des "phrases» du "Prologue» désignent la version soit démultipliée laccords complexes obtenus par multiplication harmonique, densification rythmique) ou simplifiée (réduite aux seules notes génératrices, par exemple) de ces "phrases". En regard de chaque segment ou phrase est donnée la famille harmonique ou l'accord de base pertinent la à $i$ et 1 à 41 , respectivement). Le tableau permettra de remarquer, entre autres, la coïncidence des "sommets" diatoniques ou consonants du "Prologue" et de Vue lfamilles e ef $f$ pour le premier, familles 28 à 33 pour la seconde).

Le tableau donne aussi en parallèle toutes les autres citations (plus ou moins transformées) qui doivent servir dans la polémique musicale organisée par Pousseur, et que nous examinerons maintenant. 
Tableau 3 : tableau synoptique de La Seconde Apothéose de Rameau

\begin{tabular}{|c|c|c|c|c|c|c|}
\hline \multirow[t]{2}{*}{ Mes. } & \multicolumn{4}{|c|}{$\begin{array}{c}\text { Pousseur (ange novice principal) : } \\
\text { auto-citations }\end{array}$} & \multirow{2}{*}{$\begin{array}{c}\text { Anges novices; archanges, } \\
\text { séraphins; bienheureux: } \\
\text { citations }\end{array}$} & \multirow[t]{2}{*}{ Autres } \\
\hline & & & & & & \\
\hline 1 & & & & & Boulez & \\
\hline $1-6$ & $A-$ & $a$ & & & & \\
\hline $7-8$ & & & & & & Solo (bois) série Pousseur, sons 1-5 \\
\hline 8 & & & & & Boulez (doublures en $5^{\text {tes }}$ ef $4^{\text {tes }}$ ) & \\
\hline $8-13$ & A+ & $a$ & & & & \\
\hline 14 & & & & & & Solo (vents gr.) Pousseur 1-4 \\
\hline $15-17$ & & & & & Webern m. 1-3 & \\
\hline $17-21$ & & & 1 & 1 & & \\
\hline $21-23$ & & & & & Webern m. 10-12 (trp. +2) & \\
\hline 24 & & & & & Boulez +6 & \\
\hline $24-25$ & $B-$ & $a$ & & & & \\
\hline 26 & & & & & & Solo (cl. b) dével. interv. Boulez \\
\hline $27-28$ & B & $a$ & & & & \\
\hline 29 & & & & & Stockhausen m. 160 (trp. -13 ) & \\
\hline $30-31$ & $C(+1$ & $a$ & & & & \\
\hline 32 & & & & & & Solo (fl.) Webern renv. 1-7 \\
\hline $33-34$ & & & & & Webern m. 1-3 série III & \\
\hline $35-38$ & & & 2 & $\|$ & & \\
\hline 38 & & & & & & Solo (cl.) Webern III 1-6 \\
\hline $39-40$ & & & & & Webern $m$. 10-12 série III & \\
\hline 40 & & & & & Boulez (doublures $3^{\text {ces }}$ maj.) & \\
\hline 41.42 & $\mathrm{C}+$ & a & & & & \\
\hline $43-44$ & & & & & & Solo (clav.) Webern trp. 1-12 \\
\hline $45-52$ & & & 3 à 6 & III & & \\
\hline $53-55$ & & & & & & "Solo" (2 bsns) Webern+W III \\
\hline $56-57$ & D+ & $b$ & & & & \\
\hline 58 & & & & & & Solo (tba) Webern III 6-12 \\
\hline $59-60$ & & & & & Webern $\mathrm{m} .10-12$ rétrogr. & \\
\hline $61-67$ & & & 7 & IV & & \\
\hline $68-69$ & & & & & Webern m. 1-3 rétrogr. & \\
\hline 70 & & & & & + Boulez (doublures en accord de 3 sons) & Solo (cl.) Webern III 1-6 \\
\hline 71 & $D(+)$ & $b$ & & & & \\
\hline
\end{tabular}


Tableau 3 : tableau synoptique de La Seconde Apothéose de Rameau (suite 1)

\begin{tabular}{|c|c|c|c|c|c|c|}
\hline \multirow[t]{2}{*}{ Mes. } & \multicolumn{4}{|c|}{$\begin{array}{c}\text { Pousseur (ange novice principal) : } \\
\text { auto-citations }\end{array}$} & \multirow{2}{*}{$\begin{array}{c}\text { Anges novices; archanges, } \\
\text { séraphins; bienheureux: } \\
\text { citations }\end{array}$} & \multirow[t]{2}{*}{ Autres } \\
\hline & & & Vu & & & \\
\hline 72 & & & & & Stockhausen m. 159 - hauteurs modifiées & \\
\hline 73-75 & $E+$ & $b$ & & & & \\
\hline 76 & & & & & & Solo Webern et Webern III \\
\hline 77.78 & & & & & Webern m. 10-12 série III rétrogr. (3ces!) & \\
\hline 78-84 & & & 8 à 11 & V & & \\
\hline $85-86$ & & & & & 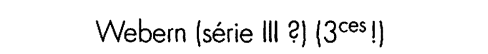 & \\
\hline 87 & & & & & & Solo (origine ?) \\
\hline $88-90$ & $E(+)$ & $b$ & & & & \\
\hline 91 & & & & & Stockhausen m. 154-157 (g. par tons!) & \\
\hline $92-93$ & $\mathrm{~F}_{+}$ & $b$ & & & & \\
\hline $94-95$ & & & & & & Solo (htb. bsn) Webern III \\
\hline $96-102$ & & & $12-13$ & $\mathrm{VI}$ & & \\
\hline $103-104$ & & & & & & Solo (clav.) Webern III 1-6 + 1-12 \\
\hline $105-106$ & $F-$ & $b$ & & & & \\
\hline 107 & & & & & & Solo (pno) \\
\hline 108-109 & G & a & & & & \\
\hline $110-111$ & & & & & Webern (annonçant la valse) (série ?̧) & \\
\hline $112-123$ & & & & & & Parallélisme accord maj. \\
\hline $124-129$ & & & $14-15$ & $\mathrm{VII}$ & & \\
\hline $130-133$ & & & & & à la Stravinsky: 9 sons de W III & \\
\hline $134-143$ & & & 16 à 18 & VIII & & \\
\hline $144-151$ & & & & & & Parall. sur cycles $5^{\text {tes }}$ partiels \\
\hline $152-154$ & & & & & Webern (annonçant la valse) & \\
\hline $155-156$ & $G-$ & a & & & & \\
\hline 157 & & & & & & Solo (pno) : g. par tons \\
\hline $158-169$ & $H$ & $c \cdot d$ & & & & \\
\hline 170 & & & & & & Claviers : transition \\
\hline 171.173 & & & 19 & IX & & \\
\hline $174-175$ & & & & & Stravinsky (6 sons) & \\
\hline $176-177$ & & & 20 & $x$ & & \\
\hline $178-181$ & & & & & Schönberg (valse dodécaphonique) & \\
\hline 182 & & & 21 & $X I$ & & \\
\hline
\end{tabular}


Tableau 3 : tableau synoptique de La Seconde Apothéose de Rameau (suite 2)

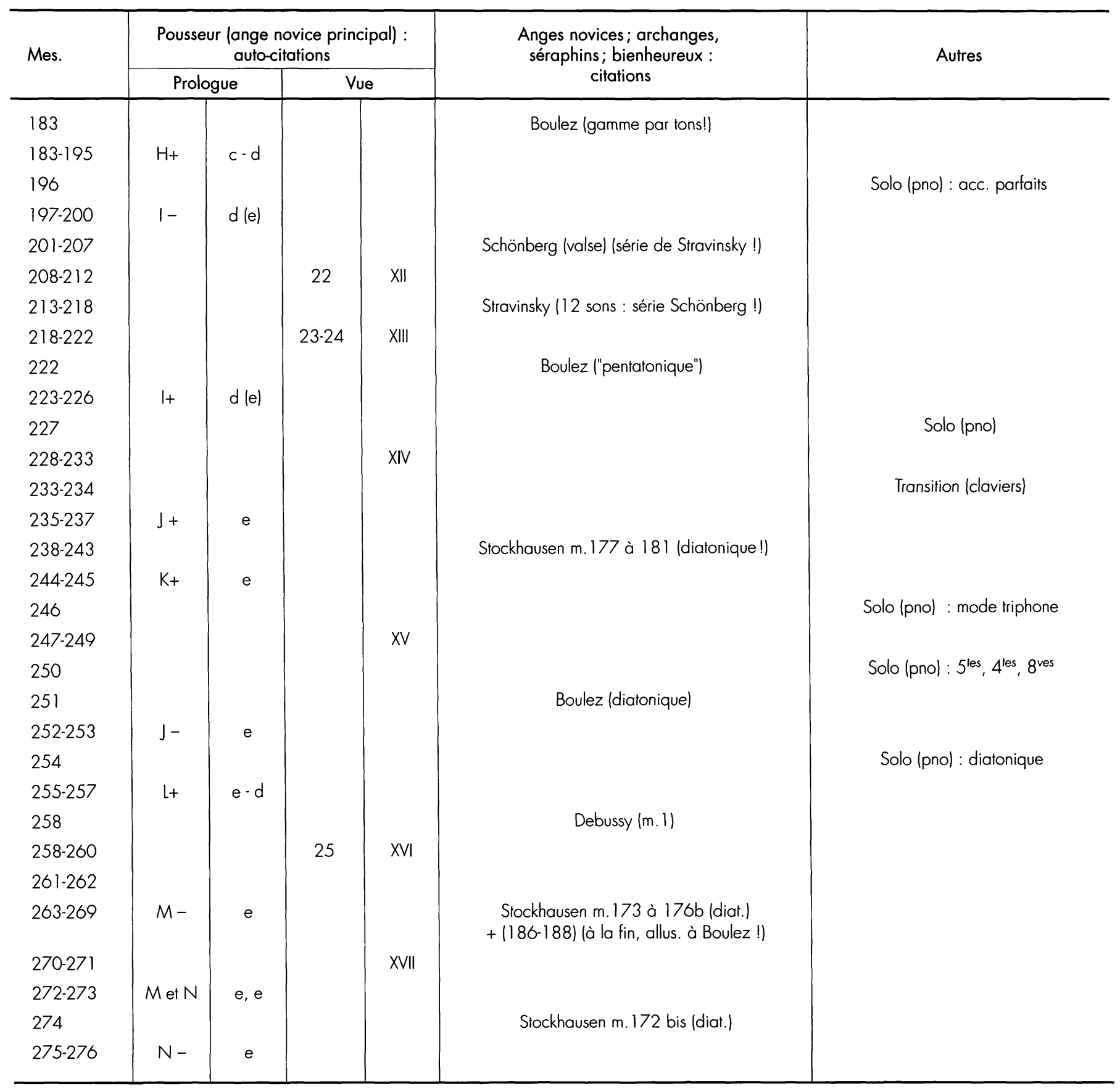


Tableau 3 : tableau synoptique de La Seconde Apothéose de Rameau (suite 3)

\begin{tabular}{|c|c|c|c|c|c|c|}
\hline \multirow{3}{*}{$\begin{array}{l}\text { Mes. } \\
277\end{array}$} & \multicolumn{4}{|c|}{$\begin{array}{l}\text { Pousseur (ange novice principal) : } \\
\text { auto-citations }\end{array}$} & \multirow{2}{*}{$\begin{array}{c}\text { Anges novices; archanges, } \\
\text { séraphins; bienheureux : } \\
\text { citations }\end{array}$} & \multirow[t]{2}{*}{ Autres } \\
\hline & \multicolumn{2}{|c|}{ Prologue } & \multicolumn{2}{|c|}{ Vue } & & \\
\hline & $M+$ & e & & & & \\
\hline $278-280$ & & & 26 & $X V I I I$ & & \\
\hline $281-283$ & $O-$ & $f$ & & & & \\
\hline $284-285$ & & & & & Debussy (m.3-4) & \\
\hline $286-292$ & & & 27 & $X \mid X$ & à 286 et $290:$ annonce de Scheidt & \\
\hline $293-298$ & $\mathrm{~N}$ et $\mathrm{O}$ & $e, f$ & & & à 293 : Bartholomée & \\
\hline 298 & & & & & Debussy (m. 1) & \\
\hline 299-308 & $P(+)$ & $f(c)$ & & & à 299 : Bartholomée & \\
\hline $309-324$ & & & 28 à 33 & $X X$ & $\begin{array}{l}\text { à } 311 \text { et } 314 \text { : annonce de Sche } \\
\text { Aussi : à } 309: \text { Bartholomée }\end{array}$ & $\begin{array}{l}320,323-324: \text { Scheidt. } \\
\text { Ne shall overcome...) }\end{array}$ \\
\hline $325-326$ & $Q \mid+1$ & $a-b$ & & & & \\
\hline $327-333$ & & & $32(33)$ & $X X I$ & Scheidt (de 328 à 332 : Scheidt varié) & \\
\hline $334-341$ & & & & & Rameau & \\
\hline $341-342$ & & & $32(33)$ & $X X \|$ & Scheidt & \\
\hline $343-346$ & & & & & Rameau & \\
\hline 347 & & & $32(33)$ & $X X \mid 11$ & Scheidt & \\
\hline 348 & & & & & Debussy m.5 & \\
\hline $349-352$ & & & & & Rameau & \\
\hline 353 & & & $32(33)$ & XXIV & Scheidt & \\
\hline $354-360$ & & & & & Rameau (avec Berio !) & \\
\hline 361 & & & $32(33)$ & $X X V$ & Scheidt & \\
\hline $362-363$ & & & & & Debussy m.5-6 & \\
\hline $364-365$ & & & $32(33)$ & $X X V I$ & Scheidt & \\
\hline $366-374$ & & & & & Rameau & \\
\hline 375 & & & $32|33|$ & $X X V \|$ & Scheidt & \\
\hline $376-389$ & $P+$ & $f(c)$ & & & & \\
\hline 390 & & & 34 & $X X V I I I$ & & \\
\hline $391-392$ & $\mathrm{R}$ & $b-g$ & & & & \\
\hline $393-396$ & & & & & Debussy m.51-54 (climax) & \\
\hline 397 & & & $32(33)$ & $X X I X$ & Scheidt : cadence (souvenir) & \\
\hline 398 & & & & & & Improvisation (série harmon.) \\
\hline $399-401$ & & & 35 & $X X X$ & à 400 : Scheidt cadence (souvenir) & \\
\hline
\end{tabular}


Tableau 3 : tableau synoptique de La Seconde Apothéose de Rameau (suite 4)

\begin{tabular}{|c|c|c|c|c|c|c|}
\hline \multirow{3}{*}{$\begin{array}{l}\text { Mes. } \\
\text { 402-404 }\end{array}$} & \multicolumn{4}{|c|}{$\begin{array}{c}\text { Pousseur (ange novice principal) : } \\
\text { auto-citations }\end{array}$} & \multirow{2}{*}{$\begin{array}{c}\text { Anges novices; archanges, } \\
\text { séraphins; bienheureux: } \\
\text { citations }\end{array}$} & \multirow[t]{2}{*}{ Autres } \\
\hline & \multicolumn{2}{|c|}{ Prologue } & \multicolumn{2}{|c|}{ Vue } & & \\
\hline & $Q_{+}$ & $a \cdot b$ & & & & \\
\hline $405-406$ & & & & & Debussy transformé & \\
\hline $407-409$ & R+ & $b-g$ & & & & \\
\hline $410-415$ & & & & $X X X \mid$ & & \\
\hline $416-418$ & & & & & & sur harmoniques naturels \\
\hline $419-423$ & & & 36 & XXXII & (cad. transformée) à 423 : Bartholomée & \\
\hline $424-425$ & $S+($ déb.) & $g(x)$ & & & & \\
\hline $426-429$ & & & & & Rameau transformé (majeur-mineur) & \\
\hline $430-431$ & $S+(f i n)$ & $g(x)$ & & & & avec improv. à 430 \\
\hline $432-435$ & & & & $X X X I I$ & & \\
\hline $436-437$ & $S($ déb.) & $g(x)$ & & & & \\
\hline 438 & & & & & Improv. sur Debussy transformé & puis improv. libre \\
\hline $439-440$ & $S($ fin $)$ & $g(x)$ & & & & \\
\hline $441-442$ & & & $37-38$ & XXXIV & & \\
\hline $443-444$ & $T$ & (i) $h$ & & & & (rythm. libre) \\
\hline 445 & & & & & & Solo (hpe) : 3 sons g. par tons \\
\hline 446 & & & & $x X X V$ & & \\
\hline $447-448$ & $T-$ & (i) $h$ & & & & \\
\hline $449-452$ & & & & $X X X V I$ & & \\
\hline $453-456$ & $U$ et $V$ & $h, h(i)$ & & & & (rythm. libre) \\
\hline $457-462$ & & & 39 & $X X X V I I$ & & \\
\hline $463-466$ & & & & & Berio & avec improv. à la fin \\
\hline $466-467$ & $u$ & h & & & & (rythm. libre) \\
\hline 467 & & & & & & We shall overcome \\
\hline $468-470$ & & & 40 & $X X X V I I I$ & & \\
\hline $471-475$ & $V-, W+(d)$. & (h) $i, i$ & & & & (rythm. libre) \\
\hline $476-478$ & & & & & & Impro (rappels) \\
\hline $479-482$ & $W+($ fin $)$ & $\mathrm{i}$ & & & & (rythm. libre) \\
\hline $483-484$ & & & & XXXIX & & \\
\hline 484 & & & & & Boulez (g. par tons) & \\
\hline $485-488$ & W- & i & & & & \\
\hline 489 & & & & & Boulez (doublures à la $3^{\text {ce }}$ maj) & \\
\hline $489-499$ & & & 41 & $L X$ & à 496 : Boulez (= accord final de Vuel & \\
\hline 500 & & & & & & Clausule (marimba) \\
\hline
\end{tabular}




\section{La musique des archanges et séraphins (Webern, Schönberg, Stravinsky)}

Parmi les quelques vénérables prédécesseurs que Pousseur cite à l'appui de sa mise en scène, Webern lqui a déjà beaucoup donné, mais d'une façon plus abstraite et éloignée, dans le cas du "Prologue»/ glisse épisodiquement ses courts fragments provenant soit des mesures 1 à 3 ou 10 à 12, qui respectivement commencent ou terminent la première section du premier mouvement de la Cantate $n^{\circ}$ 2. Ces fragments sont présentées dans leurs formes originale, renversée ou rétrogradée (les deux fragments présentent d'ailleurs de telles relations entre eux, rétrogradés même sur le plan rythmiquel, ou encore modulée selon la série Webern III dont on a parlé pour le "Prologue", "diatonisant» ses harmonies initiales (mes. 33-34), les énonçant même en tierces (mes. 77-78) (exemple 8).

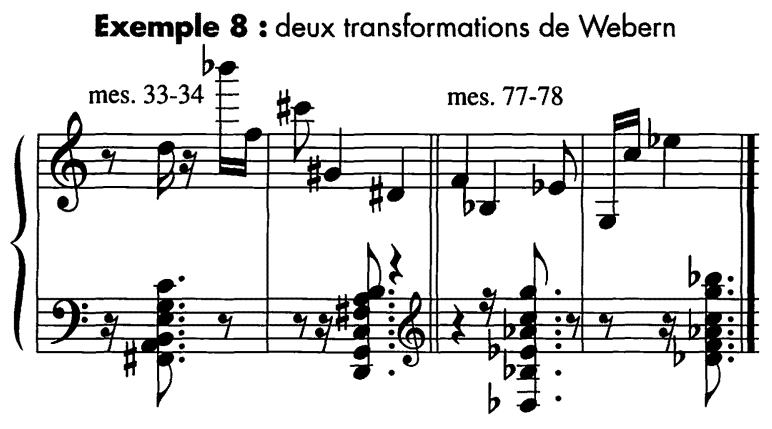

Dans ses dernières apparitions ${ }^{15}$, la citation de Webern se rapproche de l'articulation qui caractérise celle de Schönberg. À ce moment commence d'ailleurs un intéressant jeu d'influences (fussent-elles plutôt abstraites et d'ordre combinatoire) entre les citations de ces deux compositeurs et celles de Stravinsky. Pousseur ne pouvait manquer de choisir cet extrait du premier mouvement du Concerto pour piano de Schönberg à cause de son caractère de valse viennoise (dodécaphonique, comme il se doit). Mais sa deuxième apparition (mes. 201-207) énoncera non pas la série de Schönberg mais plutôt celle de Stravinsky. Si l'on suit maintenant les trois interventions dites "de Stravinsky", elles énonceront, dans ce même style tranquillo, quasi organo, qui renvoie clairement au troisième mouvement du Canticum sacrum, d'abord neuf sons de la série Webern III, lalors même que Webern se "valsifie»!); puis six sons de la série originale des premières mesures du mouvement en question du Canticum, et enfin, alors que la valse schönbergienne est passée, la série originale de cette valse.

15... dont l'origine sérielle nous échappe encore... 


\section{La musique des anges novices: Boulez, Stockhausen, Berio, Bartholomée}

Contrairement à celle de Webern, les citations-allusions à Schönberg et Stravinsky restent en dehors de la progression harmonique générale vers le diatonisme et la consonance. Elles sont encore logées dans des zones plus ambiguës sur ce plan, comme le sont d'ailleurs leurs séries constitutives. C'est stylistiquement, cependant, que leur contribution au mouvement vers le moment "tabernaculaire" à venir est le plus pertinent. Webern aura donc été celui qui, quoique encore de loin, aura servi de caution à cette progression sur le plan le plus fondamental (l'harmonie). Mais c'est (ironie!) Boulez et Stockhausen qui l'assumeront et la vivront le plus clairement, en parallèle avec la démarche que le super-compositeur qui les manipule exprime à travers ses autocitations...

Pour «représenter " Boulez, Pousseur a choisi un court motif assez prégnant issu de la Deuxième Sonate pour piano, celui qui en ouvre le troisième mouvement ${ }^{16}$. L'exemple 9 donne quelques versions de ce motif et montre les transformations qu'il subit (mes. 183, 251 et 484), malheureusement sans le contexte qui l'entoure et qui amplifie cette métamorphose... L'ironie vaut la peine d'être soulignée (même si elle préfère par essence la discrétion...): Boulez en devient, à 251, presque tendre...

Exemple 9 : versions et transformations du motif de Boulez

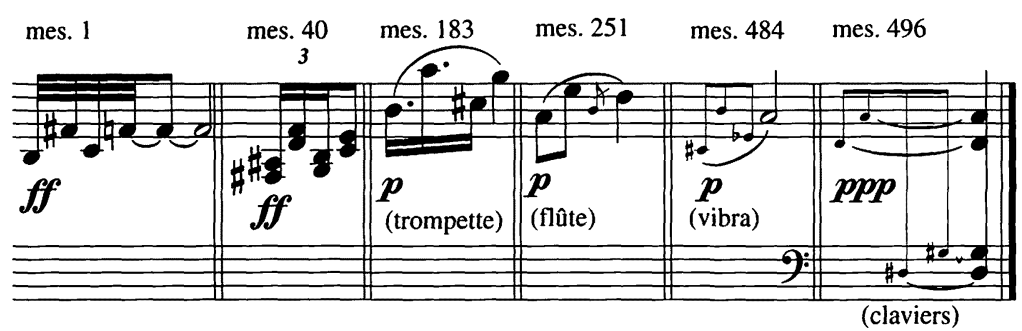

Pour ce qui est des cinq emprunts à Stockhausen, extraits d'une à quelques mesures tirés des Zeitmaße et caractérisés par une écriture pointilliste, à la rythmique parfois complexe (tempi superposés), qui dans sa plus grande densité devient "statistique", on évolue de la citation intégrale, simplement transposée là la mes. 29), à une mutation assez radicale sur le plan harmonique : à 72, modification des hauteurs de façon à articuler un réseau de quartes et tritons superposés; à 91, tout l'extrait est dans l'une des deux transpositions de la gamme par tons (!) ; à 238-243, le passage peut se subdiviser en trois fragments dont toutes les notes font partie de modes heptatoniques diatoniques (!!), par ailleurs très près
16. La présentation initiale de ce motif dans l'Apothéose, fortissimo et rapide, en fait plutôt un croisement entre le motif initial du troisième mouvement de la Sonate lintervalles identiques, mais piano staccato et scherzando) et le motif initial du premier mouvement (fortissimo et rapide, mais dont l'intervalle de quarte est projetée une octave plus bas). 
I'un de l'autre; et enfin, à 263-269, l'expression du diatonisme passe par de nombreux sauts d'octaves (!!!). L'écriture rythmique étant totalement conservée, la métamorphose de Stockhausen prend presque l'allure d'une démonstration, ou d'une conversion...

Les deux citations de Berio sont plus respectueuses de la lettre de leur auteur mais n'en recèle pas moins leur potentiel de significations, dans le cadre de la présente "polémique amicale». Celle de 463-466, jovée à la trompette accompagnée par le piano, provient du début de Coro et son caractère alangui, presque jazz, s'intègre très bien à ce moment de la courbe formelle descendante où elle s'insère. L'autre citation est plus ambiguë. Elle est jouée par la petite trompette à 354-360, comme contrechant à la citation de Rameau, et il s'agit en fait d'une mélodie belgo-francophone (Rossignolet du bois) que Pousseur avait appris à Luciano Berio (et Cathy Berberian) et qui s'est retrouvée dans les Folksongs de ce compositeur. Il est possible que ces deux citations veuillent signifier la proximité de la musique empruntée (mais non transformée) avec celle qui l'accueille, ou encore la sympathie de l'attitude de Berio, ressentie comme plus grande par Pousseur à l'endroit de sa démarche et de sa propre musique que de la part de ses deux autres collègues ${ }^{17}$.

L'emprunt de Bartholomée, joué par la harpe, est tiré de Fancy, une œuvre écrite pour ce même instrument, et adapte sans heurt au contexte du "Prologue " ou de Vue ses harmonies bâties sur un mode tétraphone majeur (3-2-3) ou « mineur» (4-1-3).
17. On en voudra pour preuve l'article de 1969 de Berio sur une représentation de Votre Faust à la Scala de Milan, où l'auteur, quoique critique pour maints aspects de l'œuvre /principalement le texte et la mise en scènel, voyait en la partition de Pousseur " une étape importante de l'histoire de la pensée musicale», "l'une des expériences les plus fertiles de notre temps 》 (Berio, 1969, p. 51-561.

\section{Le «moment tabernaculaire»: le vieil et saint ermite (Rameau) avec l'organiste allemand (Scheidt), et la musique d'un bienheureux (Debussy).}

Annoncé trois fois par Debussy lles mesures initiales de l'Hommage à Rameau extrait du premier cahier d'Images pour le piano), le couple Scheidt-Rameau entre donc enfin en scène, alors que les musiques du "Prologue" et de Vue ont rejoint les familles harmoniques susceptibles de les laisser s'y insérer (familles $f d$ 'une part, 32 et 33 d'autre part $^{18}$ ). La musique qui évoque l'auguste théoricien et compositeur français est une gavotte tirée de la Suite $n^{\circ} 1$ du cahier de Pièces de clavecin de 1731, que Pousseur a transformée en sarabande. Ses phrases sont alternées avec celles de la citation de Scheidt dans Vue, et accompagnée de deux salutations de Debussy (provenant des mesures 5 et 6 du même morceau).
18. Si l'on excepte le petit retour en arrière (325-326), par ailleurs pianissimo, comme un reste flou (familles a et $b$ ). 


\section{... et quelques autres emprunts...}

La progression "ascendante" vers ce moment "tabernaculaire" aura aussi été marquée par quelques autres événements musicaux. La plupart sont de facture monodique, confiés soit à un instrument soliste ou à une succession de solistes, parfois à un duo en hétérophonie. Ces "solos" exposent d'abord des séries clés du "Prologue» ou des portions de celles-ci (celle de Pousseur ou la série Webern III). Plus loin, confiés au piano, ce seront des exemples tirés de l'article de 1968, chacun marquant comme une borne les étapes franchis de la progression harmonique générale : à 157, gamme par tons; à 227, famille d'accords où quartes, secondes majeures et leurs renversements se mêlent; à 196, accords parfaits majeurs et mineurs; à 246, mode triphone simple; à 250 , quartes, quintes et octaves organisées en progression proche du cycle des quintes; à 254 , diatonisme; à 445 , sur la "pente descendante», mode triphone extrait de la gamme par tons.

À ces "solos" s'ajoutent deux passages en parallélisme : I'un en accords majeurs $\left(4-3^{19}\right)$ annonçant sans doute, de loin, la chanson de Scheidt, à la rythmique aussi Renaissance que sa couleur harmonique; et l'autre en accords de trois sons plus weberniens (8-3 ou 6-5). La clé de la succession mélodique de ces accords nous est encore cachée, mais l'intervalle de quinte y est important dans le premier et prépondérant dans le second passage (organisé selon des tronçons successifs d'un cycle de quintes).
19. Épellation du grave vers l'aigu des intervalles d'un accord. lci : tierce majeure (4) plus tierce mineure (3).

\section{Sur la courbe du retour : autres transformations, improvisations, coïncidences et clins d'œil à I'Utopie...}

La courbe "retour» de cette grande arche formelle - retour à des mondes harmoniques plus complexes amorcé par une longue "phrase» du "Prologue» $(\mathrm{P}+)$ - comportera aussi plusieurs passages laissant un certain degré de liberté aux interprètes. Après une dernière apparition de Debussy lles mesures du point culminant de l'Hommage à Rameaul et encadrée par deux souvenirs de Scheidt (cadences suspendues), une première improvisation se développe à partir des harmoniques naturels de si bémol et de fa aux deux cors. Directement lié aux arpèges de neuvième naturelle que l'on vient justement $d$ 'entendre dans la citation de Debussy, quel plus bel hommage pouvaiton rendre à Rameau que cette inclusion du phénomène naturel qui sert de point de départ à sa théorie harmonique! 
Un autre passage (416-418), également confié à un cor solo, jouant sur les harmoniques naturels aigus, ouvre la fenêtre sur des mondes non tempérés possibles.

Dans le cadre de cette progression harmonique, Debussy et Rameau seront tous deux entendus à travers des prismes harmoniques transformants lla première phrase de Rameau en majeur-mineur!), donnant même naissance, dans le cas de Debussy, à une courte improvisation. L'écriture plus libre, ici sur le plan rythmique, se répandra également dans certaines des dernières "phrases" du "Prologue". L'improvisation la plus libre (476-478) consistera en la superposition (contrôlée quant à sa densité générale) de passages caractéristiques que les instrumentistes choisissent, en guise de rappels, dans leur partie individuelle de la Seconde Apothéose.

Il n'est pas inutile de souligner la signification que l'inclusion de ces parties plus libres peut prendre dans l'œuvre que nous étudions. Pour Pousseur, l'aléatoire, ou l'improvisation plus ou moins dirigée, est, dans le domaine de la musique, une incarnation exemplaire de l'utopie sociale et politique, où chaque être est respecté et peut prendre librement, sans la contrainte de l'assujetissement ou de la répression, une part active à l'élaboration d'un monde meilleur. Que cette utopie, à toujours garder en tête, dans le répertoire de ses rêves, apparaisse comme l'un des fruits de l'après-Rameau est aussi significatif que l'inclusion de deux petites allusions au chant intégrationniste We shall overcome (à 313 et 467), dont Pousseur s'est déjà servi comme base de développement pour ses Couleurs croisées (1967), œuvre pour orchestre dont le titre évoque aussi bien le croisement des couleurs harmoniques et instrumentales que celles des races, en plein affrontement à l'époque de la création de cette œuvre aux États-Unis.

C'est pourquoi l'on ne peut pas dire que la musique s'endort à la fin de cette fête en l'honneur de Rameau. L'accord final est suspensif, voire pensif. Le "hasard" est beau que cet accord (celui qui termine Vue sur les jardins interdits, celui qui jumelle demi-tons avec quintes et quartes) se confonde avec celuità même constitué par la verticalisation du motif initial de Boulez. La boucle de l'œuvre est ainsi "bouclée» en rapatriant l'«ange novice » incisif du début dans la suspension interrogatrice finale, regardant dans la même direction qu'Henri Pousseur...

\section{Conclusion : le plaisir de L'Apothéose}

Une partie du plaisir que l'on tire de l'audition de la Seconde Apothéose de Rameau provient de notre connaissance de la mise en œuvre de son "programme». On pourrait ajouter que plusieurs œuvres de Pousseur demandent de s'y 
plonger avec toutes les ressources de notre culture historique et de notre savoir musical pour jouir pleinement de ce qu'elles peuvent offrir. Je pense particulièrement aux œuvres qui comportent un écheveau de textes superposées (Traverser la forêt ou Dichterliebesreigentraum) dont il est très difficile de percevoir la logique à la première audition - du moins justement sur ce même plan du texte - ou encore des œuvres (comme Les Épreuves de Pierrot l'Hébreu ou Leçons d'enfer) où les références historiques sont extrêmement nombreuses et raffinées. On se plaint parfois des œuvres faciles, ou au contraire on les vante, qui se donnent tout de suite, qui sont voulues comme clairement accessibles, faites pour communiquer immédiatement..., etc. Ici, on pourrait vraiment parler d'une œuvre qui se donne d'autant plus que l'on se penche sur elle, et d'un plaisir qui se gagne, malgré que plusieurs aspects de surface puissent gagner un auditeur moins informé, moins autoconditionné. Un esthète qui contemple un vase ancien ou tel tableau le sait chargé d'histoire et sa contemplation (et le plaisir, l'émotion qu'il en tire) est tout imprégnée de cette charge. La Seconde Apothéose de Rameau fait donc partie de cette catégorie d'œuvres chargées de références historiques et pour lesquelles notre plaisir s'accroît d'autant qu'on en sait davantage sur elles. Et lorsqu'elles deviennent comme une "seconde nature", on baignera parfois purement et simplement dans le plaisir d'une interprétation lumineuse que nous aurons eu l'occasion de vivre ${ }^{20}$.

Dévoiler le "programme " annihile-til le mystère et avec lui le plaisir? Oui, si la musique n'est pour vous que sensualité des harmonies, expression des émotions, et si l'intellect ou tout ce qui touche au domaine de la connaissance ne devraient pas trop s'en mêler... Mais si le plaisir de la musique est multiple, fouille autant dans la culture que dans la nature (la part de cette dernière étant fort complexe à cerner), il se pourrait que la polémique musicale de la Seconde Apothéose de Rameau vous soutire de nombreux sourires...

Parmi les raisons extérieures à la musique qui peuvent cependant nourrir le plaisir qu'elle nous donne, ce plaisir étant "impur" et probablement bien loin de la contemplation de "pures formes", il en est qui sont d'ordre moral. La musique politique comme la musique religieuse a voulu émouvoir en se donnant, outre des qualités essentiellement artistiques, des raisons morales. Y a-til un plaisir moral comme un plaisir de la vérité ? Derrière l'argument qui soutient la polémique musicale dont nous avons essayé d'éclairer certains des ressorts, il y a pour Pousseur plus que le plaisir d'avoir raison, fût-ce dans cette mise en scène par lui organisée. II y a les profondes convictions, les raisons profondément humanistes du "refus du refus" qui ont été à la base dé la démarche du compositeur ${ }^{21}$. Et si, derrière les œuvres, nous aimons aussi, parfois, sentir les hommes et les femmes qui les ont conçues, peut-être serons-nous également émus par le visage qui se dessine derrière la musique de la Seconde Apothéose de Rameau, et que cette analyse aura peutêtre pu contribuer à esquisser.
20. ... comme ces enfants qui, ayant découvert Le Sacre du printemps par le Fantasia de Walt Disney, y deviennent familiers et, oubliant dinosaures et volcans, jouissent maintenant des subtilités de telle ou telle interprétation...

21.... et dont les autres textes de ce numéro peuvent en faire saisir un peu les nuances de l'articulation. 


\section{Bibliographie}

BERIO, L. (1969), "Notre Faust», Contrechamps, n 1, p. 51-56 |traduction d'un article paru dans Nuova Rivista Musicale Italiana, vol. 3, 1969).

DECROUPET, P. (1989), "À la recherche de l'harmonie perdue : regards analytiques sur le "Prologue dans le ciel" d'Henri Pousseur", Bruxelles, Revue belge de musicologie, Vol. XLIII, p. 87 à 100.

POUSSEUR, H. (1968), "L'Apothéose de Rameau», Paris, Revue d'esthétique, n²1, Paris, Klincksieck, p. 105-172.

POUSSEUR, H. (1981), La Seconde Apothéose de Rameau, partition, Edizioni SuviniZerboni, Milan.

POUSSEUR, H. (1961-1968), Votre Faust, partition, Universal Edition, Vienne.

POUSSEUR, H. (1973), Vue sur les jardins interdits, partition, Edizioni Suvini-Zerboni, Milan.

POUSSEUR, H., Notes de programme sur Vue sur les jardins interdits et la Seconde Apothéose de Rameau, gracieuseté du compositeur.

\section{Discographie}

POUSSEUR, H., Votre Faust. Acteurs, chanteurs et musiciens de l'Ensemble Musiques Nouvelles sous la direction d'Henri Pousseur, coffret de trois disques vinyle, Harmonia mundi 0121580-6.

POUSSEUR, H., Vue sur les jardins interdits. Quatuor de saxophones de Montréal, Société nouvelle d'enregistrement, SNE-515.

POUSSEUR, H., La Seconde Apothéose de Rameau, Ensemble Musiques Nouvelles, dir. Pierre Bartholomée. Enregistrement privé d'Henri Pousseur. 


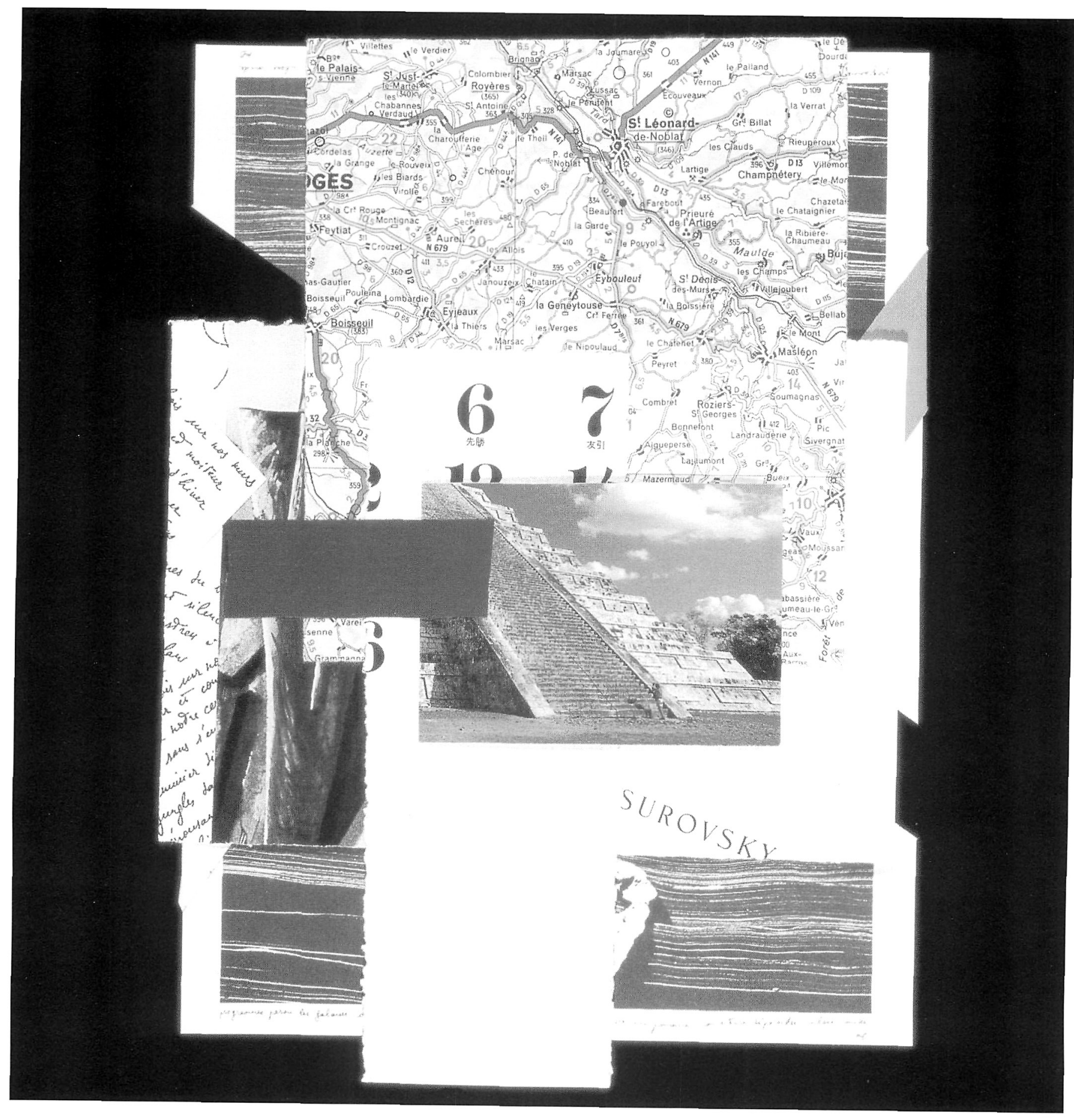

